\title{
Two-dimensional perturbations in a scalar model for shear banding
}

\author{
J.L.A. Dubbeldam ${ }^{1, a}$ and P.D. Olmsted ${ }^{2}$ \\ 1 Delft University of Technology, Mekelweg 4, 2628 CD Delft, The Netherlands \\ 2 Polymer IRC and School of Physics \& Astronomy, University of Leeds, Leeds LS2 9JT, UK
}

Received 20 March 2009 and Received in final form 8 May 2009

Published online: 31 July 2009 - (c) EDP Sciences / Società Italiana di Fisica / Springer-Verlag 2009

\begin{abstract}
We present an analytical study of a toy model for shear banding, without normal stresses, which uses a piecewise linear approximation to the flow curve (shear stress as a function of shear rate). This model exhibits multiple stationary states, one of which is linearly stable against general two-dimensional perturbations. This is in contrast to analogous results for the Johnson-Segalman model, which includes normal stresses, and which has been reported to be linearly unstable for general two-dimensional perturbations. This strongly suggests that the linear instabilities found in the Johnson-Segalman can be attributed to normal stress effects.
\end{abstract}

PACS. 47.50.-d Non-Newtonian fluid flows - 47.20.-k Flow instabilities - 47.57.Ng Polymers and polymer solutions

\section{Introduction}

Shear banding phenomena have received a lot of attention during the last decade. In shear banding the material splits into different spatial regions, or bands, that flow at different shear rates $\dot{\gamma}$. This phenomenon has been observed in granular media [1] and in viscoelastic living polymer systems such as wormlike micelle solutions [2]. Theoretically, shear banding is fairly well understood at the level of a stable one-dimensional (1D) banding profile [3-5], which connects two shear rates that are stable at a given selected total shear stress $\Sigma$. Wormlike micelles, polymer melts, and liquid crystals naturally give rise to such bistable constitutive relations [6-8]. However, the stability of bands in two or three dimensions (i.e. with respect to capillary-like fluctuations of the interface) is not well understood, despite a frequent interpretation in terms of a simple stable flat interface between coexisting states. It has been experimentally observed that the interface between the two phases is not necessarily flat, but can exhibit strong undulations and erratic fluctuations [9-11]. Theoretical calculations have shown that in shear banding flows, chaotic motion of shear bands can occur [12-14]. Such chaotic motion has also been inferred experimentally in recent works by Sood and co-workers [15-17].

A recent numerical calculation in two dimensions, which was first performed by Fielding [18] and whose results were later confirmed in refs. [18-20], demonstrated

\footnotetext{
a e-mail: j.1.a.dubbeldam@ewi.tudelft.nl
}

that, for the Johnson-Segalman model the interface between the low and high shear rate phases is linearly unstable to undulations. In this case the unstable mode involved normal stresses; however, it is still not known whether normal stresses are carried by an instability inherent in the two-dimensional (2D) nature of the fluctuation, or whether normal stresses trigger the instability [20]. Hence, in this paper we consider a simple general toy model without normal stresses $[4,14]$. We show that for a wide class of multivalued flow curves, a linear instability can never occur. Our findings imply that the coupling between convective terms and perturbations in shear stress cannot lead to a linear instability, which suggests that an instability requires other degrees of freedom, such as normal stresses.

This paper is organised as follows. In sect. 2 we introduce the model and study stationary solutions for general constitutive equations in which the shear rate is multivalued for a range of stresses. 2D perturbations are introduced in the model in sect. 3 and a linear stability analysis is carried out, from which we conclude that linear instability does not occur for our system. This strongly suggests that normal stresses are responsible for the linear instability observed in refs. [18]. The results are discussed and summarized in sect. 4 .

\section{Model description}

We consider planar Couette shear flow between flat plates separated by a distance $h$, for which the velocity field is 
$\boldsymbol{v}=\dot{\gamma} y \hat{\boldsymbol{x}}$. In the very low Reynolds number limit, which applies to the complex fluids of interest, the total shear stress $\Sigma$ is uniform in space. We assume that $\Sigma$ comprises two terms,

$$
\Sigma=\sigma_{p}(y)+\eta \dot{\gamma}(y),
$$

where the second term is the Newtonian stress of the solvent and $\sigma_{p}$ is the polymer stress. In this paper, we only consider the shear component of the stress tensor, but take the 2D nature of the perturbations in the flow field into account. Following $[14,3,4]$, we consider the following governing equation for the polymer shear stress:

$$
\left(\partial_{t}+\mathbf{v} \cdot \nabla\right) \sigma_{p}=-\frac{\sigma_{p}}{\tau}+\frac{G}{\tau} g(\dot{\gamma} \tau)+D \nabla^{2} \sigma_{p}
$$

where $\tau$ is the relaxation time of the polymer stress, $D$ is the stress diffusion coefficient, and $G$ is the plateau modulus. The function $g$ is nonmonotonic, and in refs. $[4,14]$ was taken to be

$$
g(\xi)=\frac{\xi}{1+\xi^{2}} .
$$

Stress diffusion is necessary to define a uniquely selected stress $\Sigma^{*}[5]$, as is usually found in experiments under controlled shear rate conditions. Moreover, it is natural that spatial gradients in the microstructure should be penalized, no matter how weakly.

We next make all quantities dimensionless, as noted by a carat, by expressing stress relative to $G$, length relative to the plate separation $h$, and time relative to the relaxation time $\tau$ :

$$
\begin{aligned}
& \hat{\sigma}_{p}=\sigma / G, \quad \widehat{\Sigma}=\Sigma / G, \\
& \hat{\dot{\gamma}}=\tau \dot{\gamma}, \quad \hat{D}=D \tau / h^{2}, \\
& \hat{y}=y / h, \quad \hat{x}=x / h, \\
& \hat{t}=t / \tau, \quad \hat{v}=v \tau / h .
\end{aligned}
$$

The total stress is a nonmonotonic function of shear rate $\hat{\dot{\gamma}}$. For an average shear rate $\langle\hat{\dot{\gamma}}\rangle$ imposed on the unstable portion of the flow curve, with negative slope $\partial \widehat{\Sigma} / \partial \hat{\dot{\gamma}}<0$, the system will break up into two bands, and hence a spatial variation along $\hat{y}$. Upon integrating eq. (2.1) over the vertical distance between the plates we find

$$
\widehat{\Sigma}=\left\langle\hat{\sigma}_{p}\right\rangle+\frac{1}{\alpha}\langle\hat{\dot{\gamma}}\rangle
$$

where spatial averages are defined by

$$
\langle\mathcal{O}\rangle=\int_{0}^{1} \mathcal{O}(\hat{y}) \mathrm{d} \hat{y}
$$

and we have introduced the dimensionless parameter where $\alpha \equiv G \tau / \eta$. For an inhomogeneous steady state, the polymer stress equation, eq. (2.2), leads to

$$
-\hat{\sigma}_{p}+g\left(\alpha\left(\widehat{\Sigma}-\hat{\sigma}_{p}\right)\right)+\hat{D} \hat{\sigma}_{p}^{\prime \prime}=0
$$

where the strain rate has been eliminated using eq. (2.1) and the prime denotes a $\hat{y}$-derivative, $\hat{\sigma}_{p}^{\prime}=\partial \hat{\sigma}_{p} / \partial \hat{y}$.

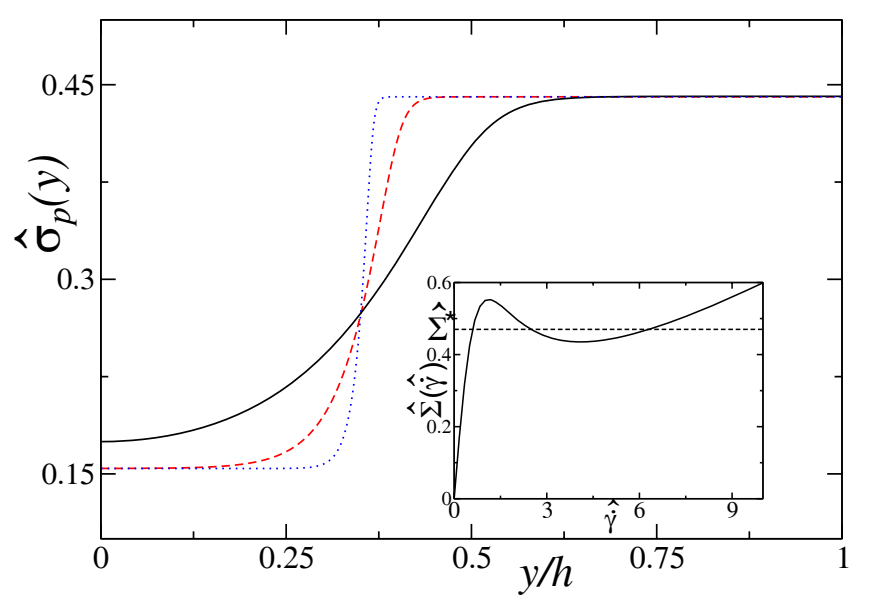

Fig. 1. The polymer stress $\sigma_{p}$ as a function of the distance $\hat{y}=y / h$ from the lower plate for $\hat{D}=0.0001$ (dotted line), $\hat{D}=0.001$ (dashed line) and $\hat{D}=0.01$ (solid line), for $\alpha=$ 20 and average shear rate $\langle\tilde{\gamma}\rangle=2.60$. The selected stress is $\widehat{\Sigma}^{*}=0.470$ for $\hat{D}=0.001$ and $\hat{D}=0.0001 ;$ and $\widehat{\Sigma}^{*}=0.471$ for $\hat{D}=0.01$. The inset shows the corresponding constitutive curve, shear stress $\widehat{\Sigma}$ as a function of $\hat{\dot{\gamma}}$, for $\alpha=20$.

As in $[18,21]$, we impose Neumann boundary conditions, $\hat{\sigma}_{p}^{\prime}(\hat{y}=0)=\hat{\sigma}_{p}^{\prime}(\hat{y}=1)=0$, for which eq. (2.10) can be solved numerically for a given stress $\widehat{\Sigma}$.

The selected stress $\widehat{\Sigma}^{*}$ is determined by the constraint of fixed average shear rate $\langle\hat{\dot{\gamma}}\rangle$. We thus eliminate $\widehat{\Sigma}$ from the stationary condition, eq. (2.10), using eq. (2.8) leading to

$$
-\hat{\sigma}_{p}+g\left[\alpha\left(\left\langle\hat{\sigma}_{p}\right\rangle-\hat{\sigma}_{p}\right)+\langle\hat{\dot{\gamma}}\rangle\right]+\hat{D} \hat{\sigma}_{p}^{\prime \prime}=0 .
$$

We numerically solve this integro-differential equation using a semi-implicit Crank-Nicolson algorithm [22]. Once a solution is found, the selected stress $\widehat{\Sigma}^{*}$ is obtained using eq. (2.8). Figure 1 shows banding profiles $\hat{\sigma}_{p}(\hat{y})$ for the function $g(\xi)$ given by eq. (2.3).

To better understand stress selection, we will generalize $g(\xi)$ to a function that allows explicit analytic calculations, but is sufficiently general to describe shear banding. The calculations discussed in the main text concern this general model. However, for comparison we also reproduce the calculations for the function $g$ defined in eq. (2.3), in appendix C. The most essential characteristic of $g$ is that it vanishes for small and large shear rates, so that the function $\widehat{\Sigma}(x)=x / \alpha+g(x)$ is nonmonotonic with a maximum and a minimum. A simple piecewise linear function showing this behavior is

$$
\widehat{\Sigma}_{0}(\hat{\dot{\gamma}})= \begin{cases}\frac{A_{c} \hat{\dot{\gamma}}}{\alpha} & \left(\hat{\dot{\gamma}}<\hat{\dot{\gamma}}_{0}\right), \\ {\left[\frac{A_{c} \hat{\dot{\gamma}}_{0}-\hat{\dot{\gamma}}_{1}}{\hat{\dot{\gamma}}_{0}-\hat{\dot{\gamma}}_{1}}\right] \frac{\left(\hat{\dot{\gamma}}-\hat{\dot{\gamma}}_{0}\right)}{\alpha}+\frac{A_{c} \hat{\dot{\gamma}}_{0}}{\alpha}} & \left(\hat{\dot{\gamma}}_{0}<\hat{\dot{\gamma}}<\hat{\dot{\gamma}}_{1}\right), \\ \frac{\dot{\hat{\gamma}}}{\alpha} & \left(\hat{\dot{\gamma}}^{2}>\hat{\dot{\gamma}}_{1}\right),\end{cases}
$$




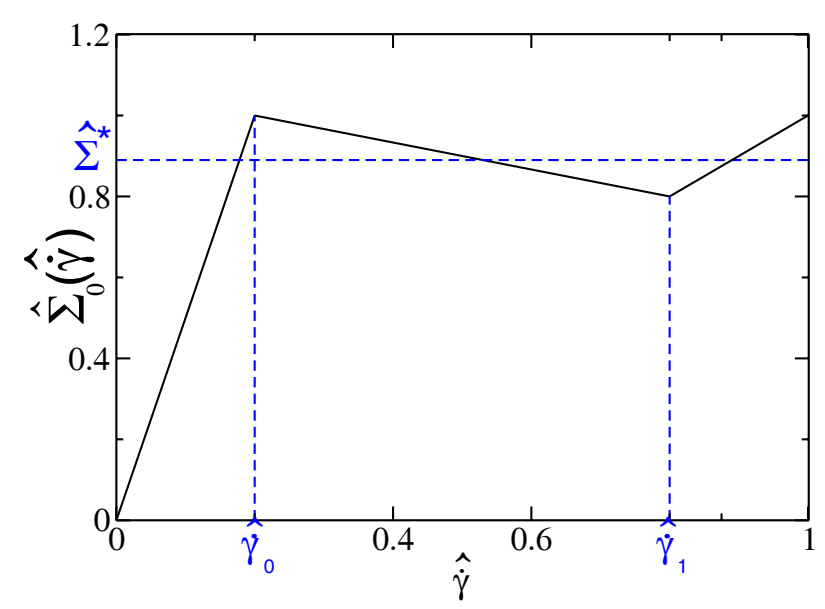

Fig. 2. The selected stress $\widehat{\Sigma}^{*}$ and the piecewise linear function $\widehat{\Sigma}_{0}(\hat{\dot{\gamma}})$ as a function of $\hat{\dot{\gamma}}$ for $\hat{\dot{\gamma}}_{0}=0.2, \hat{\dot{\gamma}}_{1}=0.8, A_{c}=5$; the selected stress is $\widehat{\Sigma}^{*}=0.891$.

where $A_{c} \hat{\dot{\gamma}}_{0}>\hat{\dot{\gamma}}_{1}$. The form of $\widehat{\Sigma}_{0}(\hat{\dot{\gamma}})$ is completely specified by the three parameters $\hat{\dot{\gamma}}_{0}, \hat{\dot{\gamma}}_{1}$, and $A_{c}$, as can be seen in fig. 2.

Using $\widehat{\Sigma}_{0}(\hat{\gamma})-\hat{\gamma} / \alpha$ instead of $g$ in the equation of motion for $\hat{\sigma}_{p}$, and eliminating $\hat{\sigma}_{p}$ using eq. (2.1), the steadystate condition for $\hat{\dot{\gamma}}$ is given by

$$
\hat{D} \hat{\gamma}^{\prime \prime}+\alpha \widehat{\Sigma}=\alpha \widehat{\Sigma}_{0}(\hat{\gamma})
$$

This is solved with Neumann boundary conditions for the stress at $\hat{y}=0$ and $\hat{y}=1$, leading to

$$
\begin{aligned}
\hat{\dot{\gamma}}_{\mathrm{I}}(\hat{y})= & c_{1} \cosh \left(\sqrt{\frac{A_{c}}{\hat{D}}} \hat{y}\right)+\alpha \frac{\hat{\Sigma}}{A_{c}} \quad\left(0<\hat{y}<\hat{y}_{0}\right), \quad(2.14 \\
\hat{\dot{\gamma}}_{\mathrm{II}}(\hat{y})= & d_{1} \cos \left(\sqrt{\frac{z}{\hat{D}} \hat{y}}\right)+d_{2} \sin \left(\sqrt{\frac{z}{\hat{D}}} \hat{y}\right) \\
& +\hat{\dot{\gamma}}_{0}-\frac{\left(A_{c} \hat{\dot{\gamma}}_{0}-\alpha \hat{\Sigma}\right)}{A_{c} \hat{\dot{\gamma}}_{0}-\hat{\dot{\gamma}}_{1}}\left(\hat{\dot{\gamma}}_{0}-\hat{\dot{\gamma}}_{1}\right) \quad\left(\hat{y}_{0}<\hat{y}<\hat{y}_{1}\right),
\end{aligned}
$$$$
\hat{\dot{\gamma}}_{\mathrm{III}}(\hat{y})=\alpha \widehat{\Sigma}+c_{2} \cosh \left(\frac{\hat{y}-1}{\sqrt{\hat{D}}}\right) \quad\left(\hat{y}_{1}<\hat{y}<1\right)
$$

where $z \equiv\left(A_{c} \hat{\dot{\gamma}}_{0}-\hat{\dot{\gamma}}_{1}\right) /\left(\hat{\dot{\gamma}}_{1}-\hat{\dot{\gamma}}_{0}\right)$, which can be recognized as the negative of the slope of the function $\widehat{\Sigma}_{0}(\hat{\gamma})$ between $\hat{\dot{\gamma}}_{0}$ and $\hat{\dot{\gamma}}_{1}$. The profile obeys the following continuity conditions:

$$
\begin{aligned}
\hat{\dot{\gamma}}_{\mathrm{I}}\left(\hat{y}_{0}\right) & =\hat{\dot{\gamma}}_{\mathrm{II}}\left(\hat{y}_{0}\right)=\hat{\dot{\gamma}}_{0}, \\
\hat{\dot{\gamma}}_{\mathrm{II}}\left(\hat{y}_{1}\right) & =\hat{\dot{\gamma}}_{\mathrm{III}}\left(\hat{y}_{1}\right)=\hat{\dot{\gamma}}_{1}, \\
\hat{\dot{\gamma}}_{\mathrm{I}}^{\prime}\left(\hat{y}_{0}\right) & =\hat{\dot{\gamma}}_{\mathrm{II}}^{\prime}\left(\hat{y}_{0}\right), \\
\hat{\dot{\gamma}}_{\mathrm{II}}^{\prime}\left(\hat{y}_{1}\right) & =\hat{\dot{\gamma}}_{\mathrm{III}}^{\prime}\left(\hat{y}_{1}\right),
\end{aligned}
$$

where again the prime denotes a $y$-derivative, $\hat{\dot{\gamma}}^{\prime}=\partial \hat{\dot{\gamma}} / \partial \hat{y}$. These six conditions guarantee a smooth solution for the shear rate and stress and from them the four constants

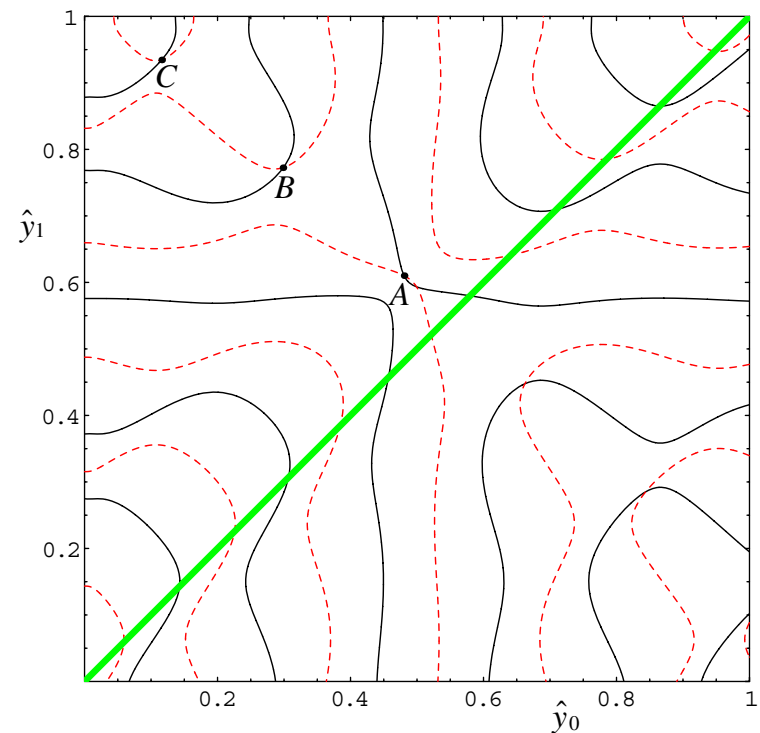

Fig. 3. The solutions $A, B, C$, for $\hat{y}_{0}$ and $\hat{y}_{1}$, for the parameter values: $A_{c}=5, \alpha=1,\langle\hat{\dot{\gamma}}\rangle=0.50, \hat{\dot{\gamma}}_{0}=0.2, \hat{\dot{\gamma}}_{1}=0.8$, and $\hat{D}=0.001$. The dashed curves correspond to eq. (2.17) and the solid ones to eq. (2.18). The physical range of solutions is in the upper left triangle, $0<y_{0}<y_{1}<1$.

$c_{1}, c_{2}, d_{1}, d_{2}$ and the locations $\hat{y}_{0}$ and $\hat{y}_{1}$ where the function $\widehat{\Sigma}_{0}(\hat{\gamma})$ switches from one piece to the next, can be determined. Appendix A contains expressions for the constants $c_{1}, c_{2}$, and $d_{1}, d_{2}$ as functions of $\hat{y}_{0}$ and $\hat{y}_{1} . d_{1}$ and $d_{2}$ are found by combining eqs. (2.15) and (2.17). Equations (2.16) and (2.18) are then used to determine the values of $\hat{y}_{0}$ and $\hat{y}_{1}$. The selected stress $\widehat{\Sigma}^{*}$ is determined by the constraint of an applied average shear rate $\langle\hat{\gamma}\rangle$, which is given by eq. (A.2). In a shear banding state the selected stress $\widehat{\Sigma}^{*}$ satisfies $\hat{\dot{\gamma}}_{1}<\widehat{\Sigma}^{*}<A_{c} \hat{\dot{\gamma}}_{0}$, and we must have $\hat{\dot{\gamma}}>0$ on the interval $\hat{y} \in[0,1]$.

A graphical solution for $\hat{y}_{0}$ and $\hat{y}_{1}$, for the case $\hat{D}=$ $0.001, A_{c}=5$ and $\langle\hat{\gamma}\rangle=0.5$, is depicted in fig. 3 , as the intersections of the contour plots of eqs. (2.17) (dashed line) and (2.18) (solid line), which should simultaneously be obeyed by $\hat{y}_{0}$ and $\hat{y}_{1}$. To solve these equations, the expressions for the constants $c_{1}, c_{2}, d_{1}, d_{2}$ from appendix A are used, eqs. (A.1), as well as expression (A.2) for the selected stress. The physical range of fig. 3 is the upper left hand triangle $0<\hat{y}_{0}<\hat{y}_{1}<1$, to the left of the diagonal line in fig. 3.

Figure 3 shows three intersection points corresponding to stationary solutions, denoted by $A=(0.4806,0.6101)$, $B=(0.2984,0.7726)$, and $C=(0.1160,0.9345)$. The values of the selected stress $\widehat{\Sigma}^{*}$ associated with these solutions are 0.8944 for $A, 0.8946$ for $B$ and 0.8949 for $C$. The fourth intersection point (the companion of $A$, with the unphysical value of $\widehat{\Sigma}^{*}=4.8$ ) is ignored, as it violates the condition $\hat{\dot{\gamma}}_{1}<\widehat{\Sigma}<A_{c} \hat{\dot{\gamma}}_{0}$.

The first solution $A$ corresponds to the common shear rate profile depicted as a solid curve in fig. 4 . The stationary solution $B$ (shown dashed) has an extra oscillation in 


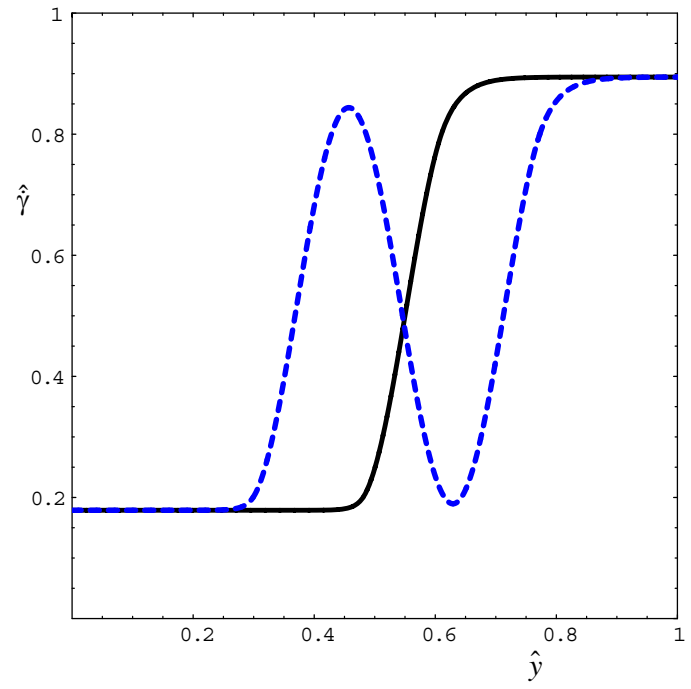

Fig. 4. Two stationary solutions corresponding to $A$ (solid curve) and $B$ (dashed curve). An additional third solution with one more oscillation is not shown.

the interface region (fig. 4), while the third solution $C$ has two oscillations (not shown). The selected stress changes monotonically as a function of an increasing number of oscillations in the resulting solution. The existence of multiple solutions for small diffusion constants is well known in the context of pattern formation in reaction-diffusion models [23]. Multiple solutions occur in our model when $\hat{D} \lesssim 10^{-2}$, with the multiplicity increasing for decreasing $\hat{D}$. Having obtained the stationary solutions, we next examine their stability by performing a linear perturbation analysis.

\section{Linear stability analysis}

\subsection{Governing equations and matching conditions}

We study the linear stability of the piecewise linear model by introducing small perturbations to the stationary $1 \mathrm{D}$ solutions,

$$
\begin{aligned}
\hat{\sigma}_{p}(\hat{x}, \hat{y}) & =\hat{\sigma}_{p}^{1 \mathrm{D}}(\hat{y})+\delta \hat{\sigma}_{p}(\hat{x}, \hat{y}), \\
\widehat{\Sigma}(\hat{x}) & =\widehat{\Sigma}^{1 \mathrm{D}}+\delta \widehat{\Sigma}(\hat{x}), \\
\hat{\dot{\gamma}}(\hat{x}, \hat{y}) & =\hat{\dot{\gamma}}^{1 \mathrm{D}}(\hat{y})+\delta \hat{\dot{\gamma}}(\hat{x}, \hat{y}), \\
\hat{\boldsymbol{v}} & =\left(\hat{v}^{1 \mathrm{D}}(\hat{y})+\delta \hat{v}_{\hat{x}}(\hat{x}, \hat{y}), \delta \hat{v}_{\hat{y}}(\hat{x}, \hat{y})\right),
\end{aligned}
$$

where the superscript ${ }^{1 \mathrm{D}}$ denotes the stationary $1 \mathrm{D}$ solution. Perturbations in the shear rate, polymer stress and total stress are related by $\delta \widehat{\Sigma}=\delta \hat{\sigma}_{p}+\hat{\dot{\gamma}} / \alpha$, which can be recast as

$$
\left\langle\delta \hat{\sigma}_{p}\right\rangle=\delta \hat{\sigma}_{p}+\delta \hat{\dot{\gamma}} / \alpha
$$

by averaging and using $\langle\delta \hat{\dot{\gamma}}\rangle=0$. Note that $\langle\delta \hat{\dot{\gamma}}\rangle=0$ implies that the average perturbation in the polymer stress $\left\langle\delta \hat{\sigma}_{p}\right\rangle$ equals the perturbation in the total stress $\delta \widehat{\Sigma}$, for $\hat{\dot{\gamma}}=\alpha\left(\delta \widehat{\Sigma}-\delta \hat{\sigma}_{p}\right)$. The shear rate is given by $\hat{\dot{\gamma}}(x, y)=$ $\partial_{\hat{y}}\left(\hat{v}^{1 \mathrm{D}}(\hat{y})+\delta \hat{v}_{x}(\hat{x}, \hat{y})\right)$, so that $\delta \hat{\dot{\gamma}}(\hat{x}, \hat{y})=\partial_{\hat{y}} \delta v_{\hat{x}}(\hat{x}, \hat{y})$. Because the flow is incompressible, a variation of the velocity in the $x$-direction must be accompanied by a compensating variation of the velocity in the $y$-direction, which obeys

$$
\partial_{\hat{y}} \delta \hat{v}_{y}(\hat{x}, \hat{y})=-\partial_{\hat{x}} \delta \hat{v}_{x}(\hat{x}, \hat{y}) .
$$

We recall that the equation of motion of the polymer stress is given by

$$
\left(\partial_{\hat{t}}+\hat{\mathbf{v}} \cdot \nabla\right) \hat{\sigma}_{p}=\widehat{\Sigma}_{0}(\hat{\dot{\gamma}})-\widehat{\Sigma}+\hat{D} \nabla^{2} \hat{\sigma}_{p}
$$

This equation can be recast in a differential equation for the shear rate $\hat{\dot{\gamma}}$, by using $\delta \hat{\sigma}_{p}=\delta \widehat{\Sigma}-\delta \hat{\dot{\gamma}} / \alpha$, from which we find to first order in all perturbations

$$
\begin{aligned}
& \partial_{\hat{t}} \delta \hat{\dot{\gamma}}+\hat{v}^{1 \mathrm{D}}(\hat{y}) \partial_{\hat{x}} \delta \hat{\dot{\gamma}}+\delta \hat{v}_{\hat{y}} \partial_{\hat{y}} \hat{\dot{\gamma}}^{1 \mathrm{D}}(\hat{y})=\left(\partial_{\hat{t}}+\hat{v}_{\hat{x}}^{1 \mathrm{D}} \partial_{\hat{x}}\right) \alpha \delta \widehat{\Sigma} \\
& +\hat{D}\left[\partial_{\hat{x} \hat{x}}+\partial_{\hat{y} \hat{y}}\right] \delta \dot{\dot{\gamma}}-\hat{D} \partial_{\hat{x} \hat{x}} \alpha \delta \widehat{\Sigma} \\
& -\alpha \widehat{\Sigma}_{0}\left(\hat{\dot{\gamma}}^{1 \mathrm{D}}+\delta \hat{\dot{\gamma}}\right)+\alpha \widehat{\Sigma}_{0}\left(\hat{\dot{\gamma}}^{1 \mathrm{D}}\right)
\end{aligned}
$$

where we explicitly kept $\widehat{\Sigma}_{0}\left(\hat{\dot{\gamma}}^{1 \mathrm{D}}+\delta \hat{\dot{\gamma}}\right)$ and $\widehat{\Sigma}_{0}\left(\hat{\dot{\gamma}}^{1 \mathrm{D}}\right)$ in order to avoid differentiating the function $\widehat{\Sigma}_{0}$ with respect to $\hat{\dot{\gamma}}$. This was done as the function $\widehat{\Sigma}_{0}$ is continuous for all $\hat{\dot{\gamma}}$, but nondifferentiable in the turning points of the stress at $\hat{\dot{\gamma}}_{0}, \hat{\dot{\gamma}}_{1}$.

Using eqs. (3.5) and (3.6) we can easily express the perturbed velocities $\delta \hat{v}_{x}(\hat{x}, \hat{y})$ and $\delta \hat{v}_{y}(\hat{x}, \hat{y})$ in terms of $\delta \hat{\dot{\gamma}}$. Starting from the identity $\frac{\partial \delta v_{\hat{x}}(\hat{x}, \hat{y}, \hat{t})}{\partial \hat{y}}=\delta \hat{\dot{\gamma}}$, we integrate from 0 to $\hat{y}$ and use the no-slip boundary condition at $\hat{y}=0$, which yields

$$
\delta \hat{v}_{x}(\hat{x}, \hat{y})=\int_{0}^{\hat{y}} \delta \hat{\dot{\gamma}}\left(\hat{x}, y^{\prime}\right) \mathrm{d} y^{\prime} .
$$

If one next differentiates $\delta \hat{v}_{x}(\hat{x}, \hat{y})$ with respect to $\hat{x}$ and substitutes the resulting expression in eq. (3.6), only one integration with respect to $\hat{y}$ (again starting from 0 and using no-slip boundary conditions) is necessary to obtain the perturbed velocity in the $\hat{y}$-direction

$$
\delta \hat{v}_{y}(\hat{x}, \hat{y})=-\int_{0}^{\hat{y}}\left[\int_{0}^{y^{\prime}} \partial_{\hat{x}} \delta \hat{\dot{\gamma}}\left(\hat{x}, y^{\prime \prime}\right) \mathrm{d} y^{\prime \prime}\right] \mathrm{d} y^{\prime} .
$$

Hence the equation for the shear rate perturbation $\delta \hat{\dot{\gamma}}(3.8)$ can be expressed entirely in terms of $\delta \hat{\dot{\gamma}}$, integrals over $\delta \hat{\dot{\gamma}}$ and $\delta \widehat{\Sigma}$. Perturbations in the shear rate must be continuous across the matching points of the bands, which are yet to be found. These follow from demanding

$$
\left.\left(\hat{\dot{\gamma}}^{1 \mathrm{D}}+\delta \hat{\dot{\gamma}}\right)\right|_{\hat{y}_{0}+\delta \hat{y}_{0}}=\widehat{\dot{\gamma}}_{0},\left.\quad\left(\hat{\dot{\gamma}}^{1 \mathrm{D}}+\delta \hat{\dot{\gamma}}\right)\right|_{\hat{y}_{1}+\delta \hat{y}_{1}}=\widehat{\dot{\gamma}}_{1},
$$

that is, the matching points are shifted by perturbations $\delta \hat{y}$ needed to bring the local shear rate to the matching points in the constitutive relation $\Sigma_{0}(\hat{\dot{\gamma}})$. Hence we split the interval $[0,1]$ into three parts: (I), $y \in\left[0, \tilde{y}_{0}\right]$; (II), $y \in\left[\tilde{y}_{0}, \tilde{y}_{1}\right]$, and (III), $y \in\left[\tilde{y}_{1}, 1\right]$, where $\tilde{y}_{i}=\hat{y}_{i}+\delta \hat{y}_{i}(\hat{x}, \hat{t})$, 
$i=1,2$. The equations of motion for the perturbations in the polymer stress are, from eq. (3.8)

$$
\begin{aligned}
\partial_{\hat{t}} \delta \hat{\dot{\gamma}}^{\mathrm{I}}= & \hat{D} \nabla^{2} \delta \hat{\dot{\gamma}}^{\mathrm{I}}-\hat{v}_{\hat{x}}^{1 \mathrm{D}}(\hat{y}) \partial_{\hat{x}} \delta \hat{\dot{\gamma}}^{\mathrm{I}}-\delta \hat{v}_{\hat{y}}(\hat{x}, \hat{y}) \partial_{\hat{y}} \hat{\dot{\gamma}}^{1 \mathrm{D}} \\
& -A_{c} \delta \hat{\dot{\gamma}}^{\mathrm{I}}+F(\alpha \delta \widehat{\Sigma}, \hat{t}), \\
\partial_{\hat{t}} \delta \hat{\dot{\gamma}}^{\mathrm{II}}= & \hat{D} \nabla^{2} \delta \hat{\dot{\gamma}}^{\mathrm{II}}-\hat{v}_{\hat{x}}^{1 \mathrm{D}}(\hat{y}) \partial_{\hat{x}} \delta \hat{\dot{\gamma}}^{\mathrm{II}}-\delta \hat{v}_{\hat{y}}(\hat{x}, \hat{y}) \partial_{\hat{y}} \hat{\dot{\gamma}}^{1 \mathrm{D}} \\
& +z \delta \hat{\dot{\gamma}}^{\mathrm{II}}+F(\alpha \delta \hat{\Sigma}, \hat{t}), \\
\partial_{\hat{t}} \delta \hat{\dot{\gamma}}^{\mathrm{III}}= & \hat{D} \nabla^{2} \delta \hat{\dot{\gamma}}^{\mathrm{II}}-\hat{v}_{\hat{x}}^{1 \mathrm{D}}(\hat{y}) \partial_{\hat{x}} \delta \hat{\dot{\gamma}}^{\mathrm{III}}-\delta \hat{v}_{\hat{y}}(\hat{x}, \hat{y}) \partial_{\hat{y}} \hat{\dot{\gamma}}^{1 \mathrm{D}} \\
& -\delta \hat{\dot{\gamma}}^{\mathrm{III}}+F(\alpha \delta \widehat{\Sigma}, \hat{t}),
\end{aligned}
$$

where we defined $F(\alpha \delta \widehat{\Sigma}, \hat{t})=\left(\partial_{\hat{t}}+\hat{v}_{\hat{x}}^{1 \mathrm{D}} \partial_{\hat{x}}-\hat{D} \partial_{\hat{x} \hat{x}}\right)$ $\alpha \delta \widehat{\Sigma}(\hat{x}, \hat{t})$. Equations (3.12) must be supplemented with boundary conditions at the matching points. At $\hat{y}=\tilde{y}_{0}$ this condition can be found from eq. (3.11),

$$
\hat{\dot{\gamma}}^{1 \mathrm{D}}\left(\hat{y}_{0}+\delta \hat{y}_{0}(\hat{x}, \hat{t})\right)+\delta \hat{\dot{\gamma}}\left(\hat{y}_{0}+\delta \hat{y}_{0}(\hat{x}, \hat{t})\right)=\hat{\dot{\gamma}}_{0}
$$

which leads, to first order in the perturbed quantities, to

$$
\delta \hat{\dot{\gamma}}\left(\hat{y}_{0}, \hat{x}, \hat{t}\right)=-\left(\frac{\partial \hat{\dot{\gamma}}^{1 \mathrm{D}}}{\partial \hat{y}}\right)_{\hat{y}_{0}} \delta \hat{y}_{0}(\hat{x}, \hat{t}) .
$$

Equation (3.14) relates the change in the shear rate to a shift in the interface position. The boundary condition at $\hat{y}=\tilde{y}_{1}$ is derived similarly. From eq. (3.14) we immediately retrieve the shift in the value of $y_{0}$, and hence the new position of the two shear bands, once $\delta \hat{\dot{\gamma}}$ is known. To deal with the inhomogeneous (time-dependent) boundary conditions in region (II) we define a new function $q(\hat{x}, \hat{y}, \hat{t})$ by

$$
\begin{aligned}
q(\hat{x}, \hat{y}, \hat{t})= & \delta \hat{\dot{\gamma}}^{\mathrm{II}}(\hat{x}, \hat{y}, \hat{t})+\frac{\left(\hat{y}-\hat{y}_{1}\right) \delta \hat{\dot{\gamma}}\left(\hat{y}_{0}, \hat{x}, \hat{t}\right)}{\hat{y}_{1}-\hat{y}_{0}} \\
& -\frac{\left(\hat{y}-\hat{y}_{0}\right) \delta \hat{\dot{\gamma}}\left(\hat{y}_{1}, \hat{x}, \hat{t}\right)}{\hat{y}_{1}-\hat{y}_{0}} \\
\equiv & \delta \hat{\dot{\gamma}}^{\mathrm{II}}(\hat{x}, \hat{y}, \hat{t})+G(\hat{x}, \hat{y}, \hat{t}),
\end{aligned}
$$

which satisfies continuity at the matching points. Hence, the function $q(\hat{x}, \hat{y}, \hat{t})$ vanishes at the boundary points by construction. In eq. (3.15) we introduced the function $G(\hat{x}, \hat{y}, \hat{t})$ for notational convenience. Using eq. (3.12b), we find the following differential equation for $q$ :

$$
\begin{aligned}
\partial_{\hat{t}} q= & z q+\hat{D} \nabla^{2} q-z G+F(\alpha \delta \widehat{\Sigma}, \hat{t})+F(G, \hat{t}) \\
& -\hat{v}_{\hat{x}}^{1 \mathrm{D}}(\hat{y}) \partial_{\hat{x}} q-\delta \hat{v}_{\hat{y}}(\hat{x}, \hat{y}) \partial_{\hat{y}} \hat{\dot{\gamma}}^{1 \mathrm{D}} .
\end{aligned}
$$

Now that we have derived the governing equations for the perturbations, we study their stability properties by employing a Fourier expansion in spatial coordinates.

\subsection{Fourier expansion}

We next expand the shear rate perturbation $\delta \hat{\dot{\gamma}}$ in Fourier modes, within the three regions, consistent with the boundary conditions $\hat{\gamma}^{\prime}=0$ at $\hat{y}=0$ and $\hat{y}=1$. This results in a continuous but nonsmooth function $\delta \hat{\dot{\gamma}}$ over the entire interval $[0,1]$ :

$$
\begin{aligned}
\delta \hat{\dot{\gamma}} \mathrm{I}(\hat{x}, \hat{y}, \hat{t})= & \sum_{n=0}^{\infty} \cos \left(\frac{\pi \hat{y}(2 n+1)}{2 \tilde{y}_{0}}\right) A_{n}(\hat{x}, \hat{t}) \\
& +\delta \hat{\dot{\gamma}}\left(\hat{y}_{0}, \hat{x}, \hat{t}\right) \quad\left(0<\hat{y}<\tilde{y}_{0}\right), \quad \\
q(\hat{x}, \hat{y}, \hat{t})= & \sum_{n=1}^{\infty} B_{n}(\hat{x}, \hat{t}) \sin \left(\frac{\pi n\left(\hat{y}-\hat{y}_{0}\right)}{\hat{y}_{1}-\hat{y}_{0}}\right) \quad\left(\tilde{y}_{0}<\hat{y}<\tilde{y}_{1}\right),
\end{aligned}
$$

$$
\begin{aligned}
\delta \hat{\dot{\gamma}}^{\mathrm{III}}(\hat{x}, \hat{y}, \hat{t})= & \sum_{n=0}^{\infty} \cos \left(\frac{\pi(\hat{y}-1)(2 n+1)}{2\left(1-\tilde{y}_{1}\right)}\right) D_{n}(\hat{x}, \hat{t}) \\
& +\delta \hat{\dot{\gamma}}\left(\hat{y}_{1}, \hat{x}, \hat{t}\right) \quad\left(\tilde{y}_{1}<\hat{y}<1\right) .
\end{aligned}
$$

Only odd cosine modes contribute in regions I and III because we require zero derivatives at the ends of the interval $[0,1]$ and a matching condition at the right, respectively left, ends of the intervals $\left[0, \tilde{y}_{0}\right]$ and $\left[\tilde{y}_{1}, 1\right]$. If one substitutes $\hat{y}=\hat{y}_{0}$ in eq. (3.17a), one finds $\delta \hat{\dot{\gamma}}^{\mathrm{I}}=\delta \hat{\dot{\gamma}}\left(\hat{y}_{0}, \hat{x}, \hat{t}\right)$, which coincides with the boundary value of $\delta \hat{\dot{\gamma}}$ in $\hat{y}_{0}$, derived above in eq. (3.11), which guarantees continuity of $\delta \hat{\dot{\gamma}}(\hat{x}, \hat{y}, \hat{t})$ throughout the interval $[0,1]$.

One can easily demonstrate that the solution of eqs. (3.17) is nonsmooth, by trying to match the derivatives at $\hat{y}=\hat{y}_{0}, \hat{y}_{1}$ using eqs. (3.17). We will not reproduce these calculation here, but only note that the narrower the interface, that is the smaller $\hat{y}_{1}-\hat{y}_{0}$, the closer the matching at the interface points $\hat{y}_{0}$ and $\hat{y}_{1}$. The piecewise linear model will in this limit approach the smooth model defined by eq. (2.3).

We therefore conclude that the perturbations are in general only continuous. To obtain an equation linking $\delta \hat{\dot{\gamma}}\left(\hat{y}_{0}, \hat{x}, \hat{t}\right)$ and $\delta \hat{\dot{\gamma}}\left(\hat{y}_{1}, \hat{x}, \hat{t}\right)$, we invoke the constraint $\langle\hat{\delta} \dot{\gamma}\rangle=0$, from which we find

$$
\begin{aligned}
& \frac{\delta \hat{\dot{\gamma}}\left(\hat{y}_{0}, \hat{x}, \hat{t}\right)}{2}\left(\hat{y}_{0}+\hat{y}_{1}\right)+\frac{\delta \hat{\dot{\gamma}}\left(\hat{y}_{1}, \hat{x}, \hat{t}\right)}{2}\left(2-\hat{y}_{1}-\hat{y}_{0}\right)= \\
& -\sum_{n=0}^{\infty} \frac{A_{n}(-1)^{n} 2 y_{0}}{(2 n+1) \pi}-\sum_{n=0}^{\infty} \frac{2\left(1-\hat{y}_{1}\right) D_{n}(-1)^{n}}{(2 n+1) \pi} \\
& -\left(\hat{y}_{1}-\hat{y}_{0}\right) \sum_{n=1}^{\infty} \frac{B_{n}\left(1-(-1)^{n}\right)}{n \pi} .
\end{aligned}
$$

Equation (3.18) constitutes the final equation needed to close the system, in a way which is similar to the method we used in sect. 2 to deduce the value of $\widehat{\Sigma}$, as we will now demonstrate.

The general idea is to find the governing equation for the amplitudes $A_{n}, B_{n}, D_{n}$ from eqs. (3.12) by projecting out the cosine (for $A_{n}$ and $D_{n}$ ) and sine modes (for $\left.B_{n}\right)$. The evolution for $\delta \hat{\dot{\gamma}}<\left(\hat{y}_{1}, \hat{x}, \hat{t}\right)$ and $\delta \hat{\dot{\gamma}}\left(\hat{y}_{0}, \hat{x}, \hat{t}\right)$ can be obtained by integrating eqs. (3.12a) and (3.12c) over their respective intervals. This is most easily done in the Fourier domain, so we expand $A_{n}(\hat{x}, \hat{t}), B_{n}(\hat{x}, \hat{t})$, $D_{n}(\hat{x}, \hat{t}), \delta \hat{\Sigma}(\hat{x}, \hat{t})$, and $\delta \hat{\dot{\gamma}}\left(\hat{y}_{0}, \hat{x}, \hat{t}\right)$ and $\delta \hat{\dot{\gamma}}\left(\hat{y}_{1}, \hat{x}, \hat{t}\right)$ in 
Fourier transforms

$$
\begin{aligned}
A_{n}(\hat{x}, \hat{t}) & =\int_{-\infty}^{\infty} a_{n}(k, \hat{t}) e^{i k \hat{x}} \mathrm{~d} k, \\
B_{n}(\hat{x}, \hat{t}) & =\int_{-\infty}^{\infty} b_{n}(k, \hat{t}) e^{i k \hat{x}} \mathrm{~d} k, \\
D_{n}(\hat{x}, \hat{t}) & =\int_{-\infty}^{\infty} d_{n}(k, \hat{t}) e^{i k \hat{x}} \mathrm{~d} k, \\
\delta \hat{\Sigma}(\hat{x}, \hat{t}) & =\int_{-\infty}^{\infty} \delta \widehat{\Sigma}(k, \hat{t}) e^{i k \hat{x}} \mathrm{~d} k, \\
\delta \hat{\dot{\gamma}}\left(\hat{y}_{0}, \hat{x}, \hat{t}\right) & =\int_{-\infty}^{\infty} \delta \hat{\dot{\gamma}}\left(\hat{y}_{0}, \hat{k}, \hat{t}\right) e^{i k \hat{x}} \mathrm{~d} k, \\
\delta \hat{\dot{\gamma}}\left(\hat{y}_{1}, \hat{x}, \hat{t}\right) & =\int_{-\infty}^{\infty} \delta \hat{\dot{\gamma}}\left(\hat{y}_{1}, \hat{k}, \hat{t}\right) e^{i k \hat{x}} \mathrm{~d} k .
\end{aligned}
$$

From eqs. (3.12a) and (3.17a) we then obtain the following evolution equation for the amplitudes $a_{n}(k, \hat{t})$ and $\delta \hat{\dot{\gamma}}\left(\hat{y}_{0}, k, \hat{t}\right)$. The amplitudes $a_{n}(k, \hat{t})$ can be extracted from this equation by projecting out the different cosine modes, whereas direct integration gives the evolution equation for $\delta \hat{\dot{\gamma}}\left(\hat{y}_{0}, k, \hat{t}\right)$. We can perform the same calculations on $\delta \hat{\dot{\gamma}}^{\mathrm{III}}$, and $q$ which leads to similar expressions involving $d_{n}(k, \hat{t}), \delta \hat{\dot{\gamma}}\left(\hat{y}_{1}, k, \hat{t}\right)$, and $b_{n}(k, \hat{t})$, which are derived in appendix $B$. It turns out that the introduction of scaled amplitudes $\left(\alpha_{n}, \beta_{n}, \delta_{n}\right)$ is beneficial for numerical and notational reasons; they are related to $\left(a_{n}, b_{n}, d_{n}\right)$ according to

$$
\begin{aligned}
\alpha_{n} & =\frac{a_{n}(-1)^{n}(2 n+1) \pi}{4}, \\
\beta_{n} & =\frac{b_{n} n \pi}{4} \\
\delta_{n} & =\frac{d_{n}(-1)^{n}(2 n+1) \pi}{4} .
\end{aligned}
$$

To show the form of the evolution equation of the amplitudes, we reproduce the governing equation for the scaled amplitudes $\alpha_{n}(k, \hat{t})$

$$
\begin{aligned}
& \frac{\mathrm{d} \alpha_{m}}{\mathrm{~d} \hat{t}}-\left(\frac{\partial \hat{\dot{\gamma}}^{1 \mathrm{D}}}{\partial \hat{y}}\right)_{\hat{y}_{0}} \partial_{\hat{t}} \delta \hat{y}_{0}(k, \hat{t})-\partial_{\hat{t}} \alpha \delta \hat{\Sigma}(k, \hat{t})= \\
& \left(A_{c}+\hat{D} k^{2}\right)\left(\frac{\partial \hat{\dot{\gamma}}^{1 \mathrm{D}}}{\partial \hat{y}}\right)_{\hat{y}_{0}} \delta \hat{y}_{0}(k, \hat{t}) \\
& -\left(A_{c}+\hat{D} k^{2}+\hat{D}\left(\frac{\pi(2 m+1)}{2 \hat{y}_{0}}\right)^{2}\right) \alpha_{m} \\
& +\hat{D} k^{2} \alpha \widehat{\Sigma}(k, \hat{t})-\frac{i k(-1)^{m}(2 m+1) \pi}{2 \hat{y}_{0}}\left[\sum_{n=1}^{\infty} \alpha_{n}(-1)^{n}\right. \\
& \times \frac{16 \hat{y}_{0}^{2}}{(2 n+1)^{3} \pi^{3}}\left[R_{m n}^{A}-T_{m}^{A}\right]+\left(\frac{\partial \hat{\dot{\gamma}}^{1 \mathrm{D}}}{\partial \hat{y}_{0}}\right)_{\hat{y}_{0}} \delta \hat{y}_{0}(k, \hat{t}) F_{m}^{A} \\
& +\sum_{n=1}^{\infty}\left[L_{m n}^{A}+\frac{D}{A_{c}} R_{m n}^{A}\right] \frac{4 \alpha_{n}(-1)^{n}}{(2 n+1) \pi}-\left(J_{m}^{A}+\frac{\hat{D}}{A_{c}} T_{m}^{A}\right) \\
& \left.\times\left[\alpha \delta \hat{\Sigma}^{A}+\left(\frac{\partial \hat{\dot{\gamma}}^{1 \mathrm{D}}}{\partial \hat{y}}\right)_{\hat{y}_{0}} \delta \hat{y}_{0}(k, \hat{t})\right]\right] \cdot
\end{aligned}
$$

The quantities $F_{m}^{A}, R_{m n}^{A}, T_{m}^{A}, L_{m n}^{A}, J_{m}^{A}$ are defined in terms of integrals over the interval $\left[0, \hat{y}_{0}\right]$. Their precise definitions can be found in appendix B, eqs. (B.3).

Finally, we need one further differential equation for the evolution of $\delta \widehat{\Sigma}(\hat{x}, \hat{t})$. This equation is found by differentiating the identity (3.18) with respect to time and subsequently substituting the expressions for $\partial_{\hat{t}} \delta \hat{\dot{\gamma}}\left(\hat{y}_{0}, k, \hat{t}\right)$ (eq. (B.2)) and $\partial_{\hat{t}} \delta \hat{\dot{\gamma}}\left(\hat{y}_{1}, k, \hat{t}\right)$ (eq. (B.5)). This yields

$$
\begin{aligned}
& \alpha \partial_{\hat{t}} \delta \widehat{\Sigma}-4\left(\hat{y}_{1}-\hat{y}_{0}\right) \sum_{n=0}^{\infty} \frac{\mathrm{d} \alpha_{n}}{\mathrm{~d} t} \frac{1}{(2 n+1)^{2} \pi^{2}}-4\left(\hat{y}_{1}-\hat{y}_{0}\right) \\
& \times \sum_{n=0}^{\infty} \frac{\mathrm{d} \delta_{n}}{\mathrm{~d} t} \frac{1}{(2 n+1)^{2} \pi^{2}}+8\left(\hat{y}_{1}-\hat{y}_{0}\right) \\
& \times \sum_{n=0}^{\infty} \frac{\mathrm{d} \beta_{2 n+1}}{\mathrm{~d} \hat{t}} \frac{1}{(2 n+1)^{2} \pi^{2}}= \\
& +4\left(1+\hat{D} k^{2}\right)\left(2-\hat{y}_{1}-\hat{y}_{0}\right) \sum_{n=0}^{\infty} \frac{\delta_{n}}{(2 n+1)^{2} \pi^{2}} \\
& +4\left(A_{c}+\hat{D}^{2}\right)\left(\hat{y}_{1}+\hat{y}_{0}\right) \sum_{n=0}^{\infty} \frac{\alpha_{n}}{(2 n+1)^{2} \pi^{2}} \\
& +\frac{\hat{D}\left(\hat{y}_{0}+\hat{y}_{1}\right)}{\hat{y}_{0}^{2}} \sum_{n=0}^{\infty} \frac{\alpha_{n}}{(2 n+1)^{2} \pi^{2}} \\
& +\frac{\hat{D}\left(2-\hat{y}_{0}-\hat{y}_{1}\right)}{\left(1-\hat{y}_{1}\right)^{2}} \sum_{n=0}^{\infty} \frac{\delta_{n}}{(2 n+1)^{2} \pi^{2}}+\left(1+\hat{D}^{2}\right) \\
& \times\left(2-\hat{y}_{1}-\hat{y}_{0}\right) \frac{\delta \hat{\gamma}_{1}}{2}+\left(A_{c}+\hat{D} k^{2}\right)\left(\hat{y}_{1}+\hat{y}_{0}\right) \frac{\delta \hat{\gamma}_{0}}{2} \\
& \alpha \hat{D} k^{2} \delta \widehat{\Sigma}^{2}+i k \Pi(k, \hat{t}),
\end{aligned}
$$

where $\Pi(k, \hat{t})$ contains the imaginary contribution to $(3.22)$, whose exact form can be found in appendix B.

To summarize, the linear stability of the shear banding states to undulating perturbation is investigated by analyzing the evolution of the amplitudes $\alpha_{n}(k, \hat{t}), \beta_{n}(k, \hat{t})$, $\delta_{n}(k, \hat{t}), \delta \hat{\Sigma}(k, \hat{t})$ and $\delta \hat{\dot{\gamma}}\left(\hat{y}_{0}, \hat{x}, \hat{t}\right), \delta \hat{\dot{\gamma}}\left(\hat{y}_{1}, \hat{x}, \hat{t}\right)$ governed by eqs. (3.21, B.6, B.4, B.2, B.5) and (3.22). The profile, and hence the interface, is stable only if all these amplitudes decay with time.

\subsection{Nonundulating perturbations for $\hat{\mathbf{D}} \rightarrow 0$}

Before turning to the general case, we will examine the limit of vanishing $\hat{D}$ and $k=0$. This special case was investigated for the Johnson-Segalman model in [14] and was also explored in [24]. The conclusion in both cases was that the shear bands in the system are neutrally stable in the absence of diffusion terms.

In sect. 2 we demonstrated how to find the stationary state(s) of system defined by eq. (2.2) graphically as the intersection point(s) of two families of curves, defined by eqs. (2.17) and (2.18). For the case $\hat{D} \rightarrow 0$, we can actually find an excellent analytic approximation to the stationary 
solution(s) which is remarkably close to stationary solutions found by the graphical method. In the limit $\hat{D} \rightarrow 0$ equations (2.16) and (2.18) reduce to

$$
\begin{aligned}
& \frac{\alpha \widehat{\Sigma}-A_{c} \hat{\dot{\gamma}}_{0}}{z} \cos \left(\sqrt{\frac{z}{\hat{D}}}\left(\hat{y}_{1}-\hat{y}_{0}\right)\right)+\left(\hat{\dot{\gamma}}_{0}-\frac{\alpha \widehat{\Sigma}}{A_{c}}\right) \sqrt{\frac{A_{c}}{z}} \\
& \times \sin \left(\sqrt{\frac{z}{\hat{D}}}\left(\hat{y}_{1}-\hat{y}_{0}\right)\right)=\left(1+\frac{\alpha \widehat{\Sigma}-A_{c} \hat{\dot{\gamma}}_{0}}{A_{c} \hat{\dot{\gamma}}_{0}-\hat{\dot{\gamma}}_{1}}\right)\left(\hat{\dot{\gamma}}_{1}-\hat{\dot{\gamma}}_{0}\right), \\
& \frac{\alpha \widehat{\Sigma}-A_{c} \hat{\dot{\gamma}}_{0}}{z} \sin \left(\sqrt{\frac{z}{\hat{D}}}\left(\hat{y}_{1}-\hat{y}_{0}\right)\right)-\sqrt{\frac{A_{c}}{z}}\left(\hat{\dot{\gamma}}_{0}-\frac{\alpha \widehat{\Sigma}}{A_{c}}\right) \\
& \times \cos \left(\sqrt{\frac{z}{\hat{D}}}\left(\hat{y}_{1}-\hat{y}_{0}\right)\right)=\frac{\left(\hat{\dot{\gamma}}_{1}-\alpha \widehat{\mathrm{s}}\right)}{\sqrt{z}},
\end{aligned}
$$

keeping all terms up to order $\sqrt{\hat{D}}$. If we next square eqs. (3.23a) and (3.23b) and add them, we find the following relation:

$$
\left(\frac{\alpha \widehat{\Sigma}-A_{c} \hat{\dot{\gamma}}_{0}}{\alpha \widehat{\Sigma}-\hat{\dot{\gamma}}_{1}}\right)^{2}=\frac{A_{c}(z+1)}{z+A_{c}} .
$$

If we would next substitute the value of $\widehat{\Sigma}$ as given by eq. (A.2), we could express $\hat{y}_{1}$ in terms of $\hat{y}_{0}$. However, first we obtain the width of the interface, that is the value of $\hat{y}_{1}-\hat{y}_{0}$, to verify if it scales proportionally to $\sqrt{\hat{D}}$ in agreement with findings in the literature. We therefore solve eqs. $(3.23)$ for $\sin \left(\sqrt{\frac{z}{D}}\left(\hat{y}_{1}-\hat{y}_{0}\right)\right)$ and obtain, after using eq. (3.24),

$$
\sin \left(\sqrt{\frac{z}{\hat{D}}}\left(\hat{y}_{1}-\hat{y}_{0}\right)\right)=\frac{\sqrt{A_{c}}+1}{\sqrt{z+1+A_{c}+A_{c} / z}} .
$$

When we invert this equation we get the following expression for interface width:

$$
\begin{aligned}
\hat{y}_{1}-\hat{y}_{0}= & \sqrt{\frac{\hat{D}}{z}}((2 M+1) \pi \\
& \left.-\arcsin \left(\frac{\sqrt{A_{c}}+1}{\sqrt{z+1+A_{c}+A_{c} / z}}\right)\right) .
\end{aligned}
$$

The other solution of eq. (3.25) is not compatible with the condition $\hat{\dot{\gamma}}_{1}<\alpha \widehat{\Sigma}<A_{c} \hat{\dot{\gamma}}_{0}$ and therefore does not correspond to a physical solution. The index $M$ is the band index. $M=0$ designates the two-band solution $(A), M=1$ has two extra bands, like solution $B$, and so on. Expression (3.26) confirms that the interface scales as $\sqrt{\hat{D}}$, as observed in $[21,14]$, and gives an exact value for the interface length as a function of $z$ and $A_{c}$. An implication of eq. (3.26) is that shear bands can only exist when $\hat{D}$ is sufficiently small. Indeed the maximum possible width of the interface is $\hat{y}_{1}-\hat{y}_{0} \simeq 1$, implying a maximum value of $\hat{D}^{*} \approx \frac{z}{(\pi-1)^{2}}$. In the case we have been considering

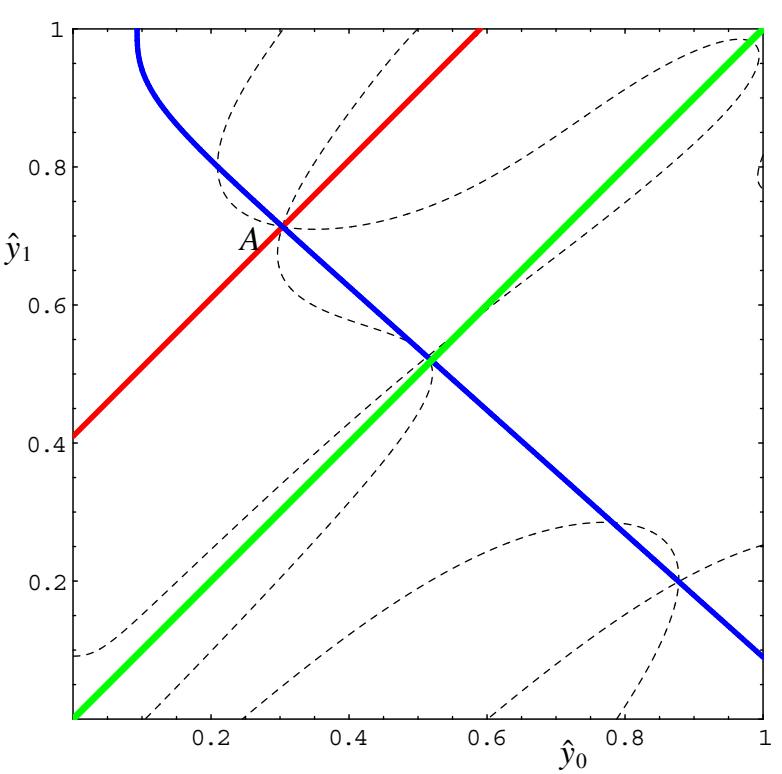

Fig. 5. Comparison of the analytical solution for $\hat{y}_{0}$ and $\hat{y}_{1}$, depicted by the intersection of the two solid curves given by eq. (3.27) and eq. (3.24), with the solution obtained by the method of sect. 2, which are printed dashed. The parameter values are $A_{c}=5.0, \hat{\gamma}_{0}=0.2, \hat{\dot{\gamma}}_{1}=0.8,\langle\hat{\dot{\gamma}}\rangle=0.5$ and $\hat{D}=0.01$. The diagonal curve indicates the line $\hat{y}_{1}=\hat{y}_{0}$ and physical solutions are in the upper left triangle. The intersection point $A$ between the two dashed curves is practically on top of the intersection point of the solid curves $\left(\hat{y}_{0}=0.3033\right.$, $\left.\hat{y}_{1}=0.7130\right)$.

$z=1 / 3$, and $A_{c}=5$, we find $\hat{D}^{*} \simeq 0.054$, which agrees with our numerical findings. The values of $\hat{y}_{0}$ and $\hat{y}_{1}$ for small $\hat{D}$ can be determined analytically by using eq. (3.24) once more, and substituting the expression of $\alpha \widehat{\Sigma}^{*}$ as given in eq. (A.2) thereby using eq. (3.26). This calculation gives the following expression for $\hat{y}_{0}$, which designates the position of the interface of the steady state with $2(M+1)$ shear bands

$$
\begin{aligned}
\hat{y}_{0}= & {\left[\frac{\left(A_{c}-1\right)\left(\sqrt{A_{c} \hat{\dot{\gamma}}_{0}}+\sqrt{\hat{\dot{\gamma}}_{1}}\right)}{\hat{\dot{\gamma}}_{0} \hat{\dot{\gamma}}_{1}}\right]\left(\left(\hat{\dot{\gamma}}_{1}-\langle\hat{\dot{\gamma}}\rangle\right) \sqrt{A_{c} \hat{\dot{\gamma}}_{0}}\right.} \\
& +\left(A_{c} \hat{\dot{\gamma}}_{0}-\langle\hat{\dot{\gamma}}\rangle\right) \sqrt{\hat{\dot{\gamma}}_{1}} \\
& \left.-\sqrt{\hat{D}}\left(1+\frac{(2 M+1) \pi-h}{\sqrt{z}}\right)\left(A_{c}-1\right)\right)
\end{aligned}
$$

where $h \equiv \arcsin \left(\frac{\sqrt{A_{c}}+1}{\sqrt{z+1+A_{c}+A_{c} / z}}\right) \simeq 0.77$ for the parameter values used in this paper. From fig. 5, one can see that the analytical solution (3.27) with $M=0$ found for $\hat{D} \rightarrow 0$, is still an excellent approximation for $\hat{D}=0.01$. The other stationary states with multiple $(2(M+1))$ bands can be found similarly, by substituting the corresponding value of $M$ in eq. (3.27). Note that multiple bands are not present for $\hat{D}=0.01$, as is shown in fig. 5 where we only find one intersection point $(A)$ of the solid curves. 
In order to study the stability of the solutions obeying eq. (3.26), to nonundulating perturbations we need to determine the eigenvalues of the evolution equations for $a_{n}(\hat{t}), b_{n}(\hat{t}), d_{n}(\hat{t})$ (or equivalently $\alpha_{n}(\hat{t}), \beta_{n}(\hat{t}), \delta_{n}(\hat{t})$ ), $\delta \hat{\dot{\gamma}}\left(\hat{y}_{0}, \hat{t}\right), \delta \hat{\dot{\gamma}}\left(\hat{y}_{0}, \hat{t}\right)$ and $\delta \widehat{\Sigma}(\hat{t})$ for $k=0$. Because the imaginary parts of the governing equations all depend linearly on $k$, we can restrict ourselves to the real part of the differential equations for the amplitudes $\alpha_{n}, \beta_{n}, \delta_{n}, \delta \hat{\dot{\gamma}}\left(\hat{y}_{0}, \hat{t}\right)$, $\delta \hat{\gamma}\left(\hat{y}_{0}, \hat{t}\right)$ and $\delta \widehat{\Sigma}$. These equations can easily be found by projecting out the different sine and cosine modes and are given by eqs. (B.1, B.6, B.4, B.2, B.5), and (B.9). For $k=0$ and $\hat{D} \rightarrow 0$ the evolution equations for $\alpha_{n}(k, \hat{t})$, $\beta_{n}(k, \hat{t}), \delta_{n}(k, \hat{t}), \delta \hat{\dot{\gamma}}\left(\hat{y}_{0}, \hat{t}\right), \delta \hat{\dot{\gamma}}\left(\hat{y}_{1}, \hat{t}\right)$ and $\delta \widehat{\Sigma}(k, \hat{t})$ read:

$$
\begin{aligned}
& \frac{\mathrm{d} \alpha_{m}}{\mathrm{~d} \hat{t}}-\frac{\mathrm{d} \alpha \delta \hat{\Sigma}}{\mathrm{d} \hat{t}}+\frac{\mathrm{d} \delta \hat{\dot{\gamma}}\left(\hat{y}_{0}, \hat{t}\right)}{\mathrm{d} \hat{t}}=-A_{c} \delta \hat{\dot{\gamma}}\left(\hat{y}_{0}, \hat{t}\right)-A_{c} \alpha_{m}, \\
& \frac{\mathrm{d} \beta_{m}}{\mathrm{~d} \hat{t}}-\frac{\mathrm{d} \alpha \delta \hat{\Sigma}}{\mathrm{d} \hat{t}}\left(\frac{1-(-1)^{m}}{2}\right)+\frac{1}{2} \frac{\mathrm{d} \delta \hat{\dot{\gamma}}\left(\hat{y}_{0}, \hat{t}\right)}{\mathrm{d} \hat{t}} \\
& -\frac{(-1)^{m}}{2} \frac{\mathrm{d} \delta \dot{\gamma}\left(\hat{y}_{1}, \hat{t}\right)}{\mathrm{d} \hat{t}}=z\left(1-\frac{m^{2} \pi^{2}}{((2 M+1) \pi-h)^{2}}\right) \beta_{m} \\
& +\frac{z}{2} \delta \hat{\dot{\gamma}}\left(\hat{y}_{0}, \hat{t}\right)-\frac{z(-1)^{m}}{2} \delta \hat{\dot{\gamma}}\left(\hat{y}_{1}, \hat{t}\right), \\
& \frac{\mathrm{d} \delta_{m}}{\mathrm{~d} \hat{t}}-\frac{\mathrm{d} \alpha \delta \hat{\Sigma}}{\mathrm{d} \hat{t}}+\frac{\mathrm{d} \delta \hat{\dot{\gamma}}\left(\hat{y}_{1}, \hat{t}\right)}{\mathrm{d} \hat{t}}=-\delta \hat{\dot{\gamma}}\left(\hat{y}_{1}, t\right)-\delta_{m}, \quad(3.28 \mathrm{c}) \\
& \frac{\mathrm{d} \delta \hat{\dot{\gamma}}\left(\hat{y}_{0}, \hat{t}\right)}{\mathrm{d} \hat{t}}-\frac{\mathrm{d} \alpha \delta \hat{\Sigma}}{\mathrm{d} \hat{t}}+\sum_{n=0}^{\infty} \frac{\mathrm{d} \alpha_{n}}{\mathrm{~d} \hat{t}} \frac{8}{(2 n+1)^{2} \pi^{2}}= \\
& -A_{c} \delta \hat{\dot{\gamma}}\left(\hat{y}_{0}, \hat{t}\right)-A_{c} \sum_{n=0}^{\infty} \frac{8 \alpha_{n}}{(2 n+1)^{2} \pi^{2}}, \\
& \frac{\mathrm{d} \delta \hat{\dot{\gamma}}\left(\hat{y}_{1}, \hat{t}\right)}{\mathrm{d} \hat{t}}-\frac{\mathrm{d} \alpha \delta \hat{\Sigma}}{\mathrm{d} \hat{t}}+\sum_{n=0}^{\infty} \frac{\mathrm{d} \delta_{n}}{\mathrm{~d} \hat{t}} \frac{8}{(2 n+1)^{2} \pi^{2}}= \\
& -\delta \hat{\dot{\gamma}}\left(\hat{y}_{1}, \hat{t}\right)-\sum_{n=0}^{\infty} \frac{8 \delta_{n}}{(2 n+1)^{2} \pi^{2}}, \\
& \frac{\mathrm{d} \alpha \delta \hat{\Sigma}}{\mathrm{d} \hat{t}}=A_{c}\left(\hat{y}_{0}+\hat{y}_{1}\right) \frac{\delta \hat{\dot{\gamma}}\left(\hat{y}_{0}, \hat{t}\right)}{2}+(28 \mathrm{e}) \\
& +4 A_{c}\left(\hat{y}_{0}+\hat{y}_{1}\right) \sum_{n=0}^{\infty} \frac{\alpha_{n}}{(2 n+1)^{2} \pi^{2}} \\
& +4\left(2-\hat{y}_{1}-\hat{y}_{0}\right) \frac{\delta \hat{\dot{\gamma}}\left(\hat{y}_{1}, \hat{t}\right)}{2} \sum_{n=0}^{\infty} \frac{\delta_{n}}{(2 n+1)^{2} \pi^{2}} .
\end{aligned}
$$

Equations (3.28) contain a lot of information. It is immediately clear, for example, that $\lambda=0$ is an eigenvalue of eqs. (3.28), by which we mean that solutions with all amplitudes decaying as $\exp (-\lambda t)$, with $\lambda=0$, exist. This can be seen by interpreting eqs. (3.28) as a matrix equation which has the eigenvalue corresponding to $\delta \widehat{\Sigma}$ equal to zero.

If it is proved that all amplitudes except $\frac{\mathrm{d} \delta \widehat{\Sigma}}{\mathrm{d} \hat{t}}=0 \mathrm{al}-$ ways decay, and thus have eigenvalues with positive real parts, we have shown that the shear-banded state is neutrally stable. This agrees with Fielding [14] and Yih [24].
It should also be noticed that the equation for $\delta \widehat{\Sigma}$ decouples from the other equations as no $\delta \widehat{\Sigma}$ terms appear in the equations for the other amplitudes and we could therefore elimate all $\partial_{\hat{t}} \delta \widehat{\Sigma}$ terms in eqs. (3.28) by substituting the right-hand side of eq. (3.28f) for $\partial_{\hat{t}} \delta \widehat{\Sigma}$ in first five equations of (3.28). Thus we only need to focus on eqs. (3.28a-3.28e).

By subtracting eqs. (3.28d) from (3.28a), we can find an equation expressed entirely in terms of $\alpha_{n}$, and subtracting eqs. (3.28e) from (3.28c) gives an equation in terms of $\delta_{n}$ :

$$
\begin{aligned}
& \frac{\mathrm{d} \alpha_{m}}{\mathrm{~d} \hat{t}}-8 \sum_{n=0}^{\infty} \frac{\mathrm{d} \alpha_{n}}{\mathrm{~d} \hat{t}} \frac{1}{(2 n+1)^{2} \pi^{2}}= \\
& -A_{c}\left[\alpha_{m}-8 \sum_{n=0}^{\infty} \frac{\alpha_{n}}{(2 n+1)^{2} \pi^{2}}\right], \\
& \frac{\mathrm{d} \delta_{m}}{\mathrm{~d} \hat{t}}-8 \sum_{n=0}^{\infty} \frac{\mathrm{d} \delta_{n}}{\mathrm{~d} \hat{t}} \frac{1}{(2 n+1)^{2} \pi^{2}}= \\
& -\left[\delta_{m}-8 \sum_{n=0}^{\infty} \frac{\delta_{n}}{(2 n+1)^{2} \pi^{2}}\right] .
\end{aligned}
$$

The eigenvalues of eq. (3.29a) are $\lambda=A_{c}$ and of eq. (3.29b) $\lambda=1$, reflecting the decay of these amplitudes in time. When we next consider eqs. (B.2) and (B.5) and eq. (3.28f) to eliminate the $\frac{\mathrm{d} \delta \widehat{\Sigma}}{\mathrm{d} \hat{t}}$ terms, we find that the perturbations in the interface shear stresses obey

$$
\begin{aligned}
& \frac{\mathrm{d} \delta \hat{\dot{\gamma}}_{0}}{\mathrm{~d} \hat{t}}=-A_{c}\left(1-\frac{\hat{y}_{0}+\hat{y}_{1}}{2}\right) \delta \hat{\dot{\gamma}}_{0}+\left(1-\frac{\hat{y}_{0}+\hat{y}_{1}}{2}\right) \delta \hat{\dot{\gamma}}_{1}, \\
& \frac{\mathrm{d} \hat{\dot{\gamma}}_{1}}{\mathrm{~d} \hat{t}}=-\left(\frac{\hat{y}_{0}+\hat{y}_{1}}{2}\right) \hat{\dot{\gamma}}_{1}+A_{c}\left(\frac{\hat{y}_{0}+\hat{y}_{1}}{2}\right) \hat{\dot{\gamma}}_{0} .
\end{aligned}
$$

Using eq. (3.18) with the amplitudes $A_{n}$ and $D_{n}$ set to zero, as we have previously found that these decay in time, and neglecting terms of order $\hat{y}_{1}-\hat{y}_{0}$, we readily find that eqs. (3.30a) and (3.30b) reduce to a single differential equation for $\hat{\dot{\gamma}}_{0}$ that reads

$$
\frac{\mathrm{d} \delta \hat{\dot{\gamma}}_{0}}{\mathrm{~d} \hat{t}}=-A_{c}\left(1-\hat{y}_{0}\right) \delta \hat{\dot{\gamma}}_{0}-\hat{y}_{0} \delta \hat{\dot{\gamma}}_{0},
$$

which clearly corresponds to a solution that decreases in time. As in this approximation $\delta \hat{\dot{\gamma}}_{1}$ is proportional to $\delta \hat{\dot{\gamma}}_{0}$ the same holds true for $\delta \hat{\dot{\gamma}}_{1}$, showing that the position of the interface is linearly stable. This means that the stability of eqs. (3.28) only depends on the properties of the amplitudes $\beta_{m}$. The even and odd modes of the amplitudes $\beta_{m}$ satisfy different evolution equations. Using the fact that $\alpha_{n}, \delta_{n}$ and $\delta \hat{\dot{\gamma}}_{0}, \delta \hat{\gamma}_{1}$ decay in time, the stability properties of the amplitudes $\beta_{n}$ for both odd and even $n$ are determined by the equation

$$
\frac{\mathrm{d} \beta_{n}}{\mathrm{~d} \hat{t}}=z\left(1-\frac{n^{2} \pi^{2}}{((2 M+1) \pi-h)^{2}}\right) \beta_{n},
$$




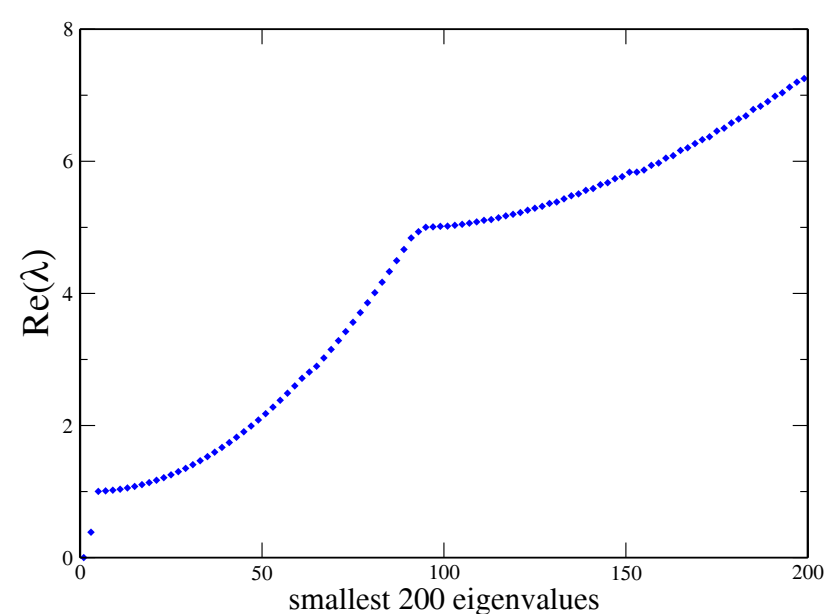

Fig. 6. Real parts of the 200 eigenvalues with smallest real parts of the system of eqs. (3.28) for nonundulating perturbations. The parameter values are $A_{c}=5, \hat{y}_{0}=0.4806$, $\hat{y}_{1}=0.6101,\langle\hat{\gamma}\rangle=0.5, \hat{\dot{\gamma}}_{0}=0.2, \hat{\dot{\gamma}}_{1}=0.8$, and $\hat{D}=10^{-5}$ The smallest eigenvalue is 0 , demonstrating that the system is neutrally stable. Eigenvalues are more concentrated around $\lambda=1$ and $\lambda=5$ in agreement with the analytical asymptotic result. The first eigenvalue $\lambda=0$ is related to $\delta \widehat{\Sigma}$, the second with $\lambda \approx 0.1$ to $\beta_{1}$ and all eigenvalues $\lambda=1$ to $\alpha_{n}$, and $\lambda=A_{c}=5$ to $\delta_{n}$.

where $M$ is the band index. The system of eqs. (3.32) gives eigenvalues

$$
\lambda_{n}=-z\left(1-\frac{n^{2} \pi^{2}}{((2 M+1) \pi-h)^{2}}\right) .
$$

As the smallest eigenvalue is $\lambda_{1}$, the sign of this quantity determines the stability of the amplitude of $\beta_{n}$ and therefore the stability of the system. For $M=0, \lambda_{1}>0$, which proves that the two-banded solution is neutrally stable: all amplitudes $\alpha_{n}, \beta_{n}, \delta_{n}, \delta \hat{\dot{\gamma}}_{0}, \delta \hat{\dot{\gamma}}_{1}$ are decaying in time and $\alpha \delta \widehat{\Sigma}$ is staying constant in time. Multiple-banded modes with $M=1,2$ all have an eigenvalue $\lambda_{1}<0$, which indicates instability of these modes.

In conclusion we have proved that for small $\hat{D}$ the stationary shear band solution with two bands is neutrally stable against non undulating perturbations and all other stationary states are unstable. Our analytical findings can be verified numerically. In fig. 6 the results of a numerical calculation of the 200 eigenvalues with smallest real part are displayed, for $\hat{D}=10^{-5}$. Besides the zero eigenvalue corresponding to $\delta \widehat{\Sigma}$, we indeed find $\lambda=A_{c}$ corresponding to the amplitudes $\alpha_{n}$, and $\lambda=1$ corresponding to the amplitudes $\delta_{n}$, many times. The degeneracy is lifted by the small, but finite value of $\hat{D}$ and the finite size of the matrix. The eigenvalue $\lambda \approx 0.1$ corresponds to $\beta_{1}$. To check our result that all multiple bands solutions are unstable, we numerically calculated the smallest eigenvalues of eqs. (3.28) for the solutions $B$ and $C$, found in sect. 2 . We indeed find that solutions $B$ and $C$ are unstable to small nonundulating perturbations. This is shown in the graph in fig. 7 , where an eigenvalue with real part smaller than zero was found for both solutions $B$ and $C$.

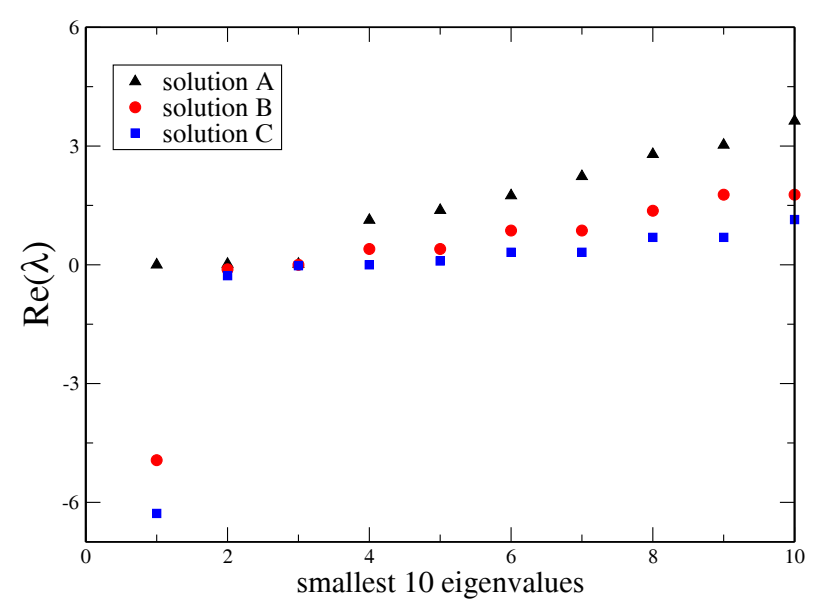

Fig. 7. The real part of the first 10 eigenvalues of $\mathbf{S}^{-1} \mathbf{L}^{\mathbf{R}}$ for an expansion of eq. (2.1) in $n=1000$ modes with zero wave number $k$ for solutions $A(\triangle), B(\circ)$, and $C(\square)$. The parameters are the same as in fig. $3: A_{c}=5,\langle\hat{\gamma}\rangle=0.50$, and $\hat{\dot{\gamma}}_{0}=0.2, \hat{\dot{\gamma}}_{1}=0.8$; the diffusion constant $\hat{D}=0.001$. It can be clearly seen that the real part of the eigenvalues for solutions $B$ and $C$ crosses zero, indicating instability of the solutions with multiple bands. Only the solution with two bands $(A)$ is (neutrally) stable.

We next turn to the general case in which the diffusion is no longer assumed to vanish and undulations in the perturbations are admitted.

\subsection{Linear stability: nonzero diffusion}

The linear stability of the stationary solutions found in sect. 2 with respect to undulatory perturbations are examined numerically. We follow the same strategy as in the previous section, projecting out the different sine and cosine modes, but this time keeping the imaginary parts of the evolution equations (3.17) as $k$ is no longer presumed to vanish.

It again proves beneficial to use the scaled amplitudes $\alpha_{n}(k, \hat{t}), \beta_{n}(k, \hat{t}), \delta_{n}(k, \hat{t})$ defined in eq. (3.20) rather than the amplitudes $a_{n}(k, \hat{t}), b_{n}(k, \hat{t}), d_{n}(k, \hat{t})$, for numerical convenience. Once we have projected out the sine and cosine modes, we are left with the evolution equations of the amplitudes. The details of this calculation are relegated to appendix B. Here we merely note that the general structure of the system of differential equations, whose stability we would like to explore, can easily be captured in a matrix equation.

By introducing a vector $\boldsymbol{u}=\left(\alpha_{0}, \cdots, \alpha_{n}, \beta_{1}, \cdots, \beta_{n}\right.$, $\left.\delta_{0}, \cdots, \delta_{n}, \delta \hat{\dot{\gamma}}_{0}, \delta \hat{\dot{\gamma}}_{1}, \delta \widehat{\Sigma}\right)$, the matrix equivalent of the amplitude evolution is given by

$$
\mathbf{S} \cdot \frac{\mathrm{d} \boldsymbol{u}}{\mathrm{d} t}=-\left(\mathbf{L}^{\mathbf{R}}-2 i k \mathbf{L}^{\mathbf{I}}\right) \cdot \boldsymbol{u}
$$

where the $(3 n+5) \times(3 n+5)$ matrices $\mathbf{S}$ and $\mathbf{L}^{\mathbf{R}}, \mathbf{L}^{\mathbf{I}}$ can be easily read off from the amplitude equations (B.1-B.4) in appendix B. As in the previous section, eigenvalues of 


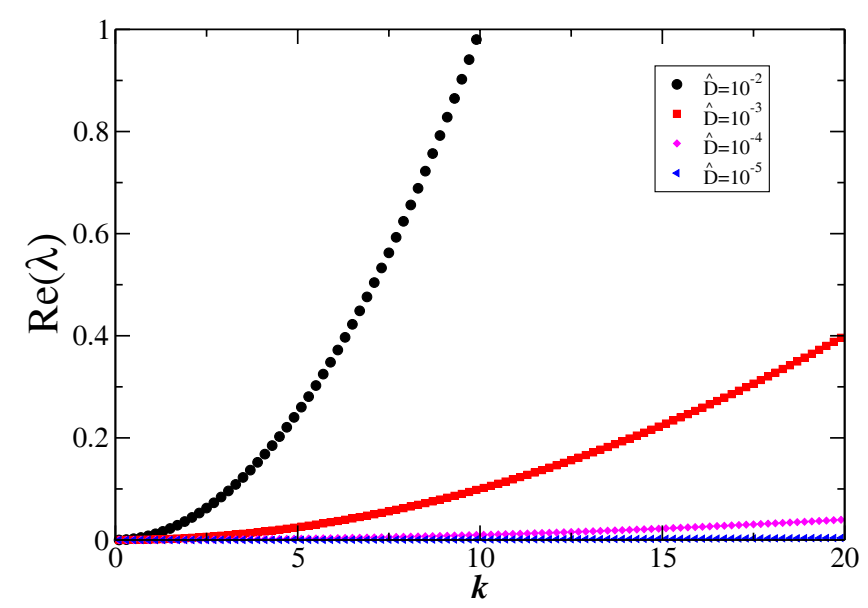

Fig. 8. The eigenvalue with smallest real part as a function of $k$ for solution $A$ using different values of the diffusion constant $\hat{D}$. The eigenvalue with smallest real part is observed to increase quadratically with $k$. Moreover, the eigenvalues decrease with decreasing $\hat{D}$, eventually approaching zero, a signal of neutral stability.

the matrix $\mathbf{S}^{-1} \tilde{\mathbf{L}} \equiv \mathbf{S}^{-1} \mathbf{L}^{\mathbf{R}}-2 i k \mathbf{S}^{-1} \mathbf{L}^{\mathbf{I}}$ with positive real part correspond to stable solutions. The linear stability of the stationary solutions found in sect. 3.3 with respect to nonundulatory perturbations is retrieved by setting $k=0$ in eq. (3.34).

The results of the numerical calculations that were performed for this special case are presented in figs. 6 and 7 show good agreement with the analytical estimate for $\hat{D} \rightarrow 0$ and $k=0$. The general case for $k \neq 0$ does not allow any tractable analytical expressions for the eigenvalues. We therefore have to resort to numerical methods.

To find the real part of eigenvalues for the stationary solution $A$, we diagonalize the matrix $\tilde{\mathbf{L}}$ numerically using the QR algorithm from ref. [22]. We verified our results with the LAPACK linear algebra package [25], and verified convergence of the eigenvalues up to $n=1000$.

Figure 8 shows the real part of the least stable eigenvalue, i.e. that with smallest real part, as a function of $k$. The dependence on $k$ is quadratic and for $k=0$ the smallest eigenvalue is equal to 0 . The stability decreases with decreasing $\hat{D}$; for $\hat{D}$ decreasing, at a fixed value of $k=10$, from $\hat{D}=10^{-2}$ to $\hat{D}=10^{-5}, \operatorname{Re}(\lambda)$ changes from 1.0 to about $10^{-3}$.

To investigate the distribution of eigenvalues, we plot the 100 eigenvalues with the smallest real parts for fixed $k=3$ in fig. 9. One can still clearly distinguish the plateaus at $\operatorname{Re}(\lambda)=1$ and $\operatorname{Re}(\lambda)=A_{c}=5$. The plateaus become smaller for increasing $D$, as the eigenvalues increase more rapidly for larger $\hat{D}$.

To verify our calculations, we have performed the same calculations for the smooth model originally introduced in eq. (2.3) (see appendix $\mathrm{C}$ for details). We find that the smooth model indeed qualitatively reproduces the numerical results of the piecewise toy model.

Moreover, for this model the shear banding state with two bands is also linearly stable with respect to undulations, as witnessed by the positivity of the real parts of

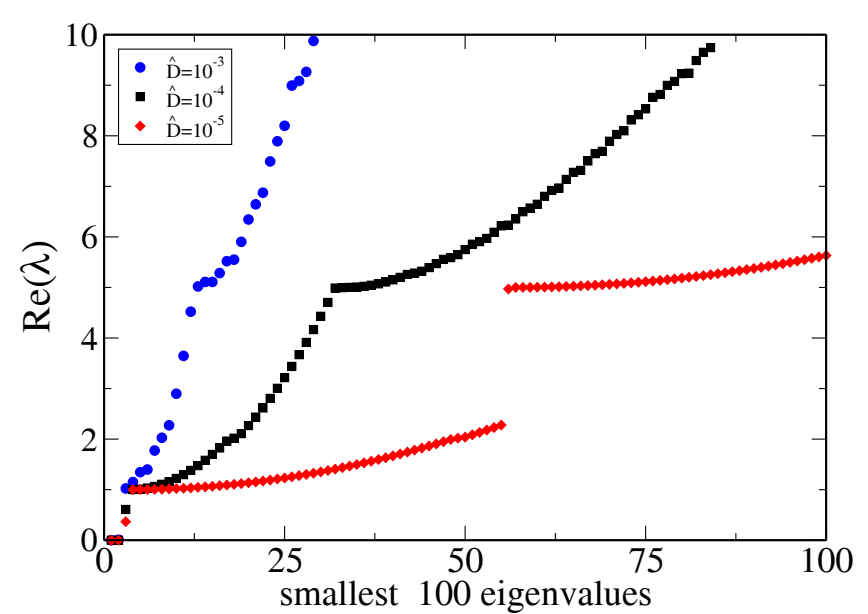

Fig. 9. The distribution of the first 100 eigenvalues with smallest real part for fixed $k=3$ and different values of the diffusion constant $\hat{D}$. The stair case is clearly recognizable and a clear accumulation of eigenvalues occurs at $\operatorname{Re}(\lambda)=1$ and $\operatorname{Re}(\lambda)=A_{c}=5$.

the eigenvalues in fig. 10. Although we report here only the results $A_{c}=5$, we verified linear stability of the twoband solution for a large number of values of $A_{c} \in[1,10]$. This agrees with the picture that arose from our analytical study for the case of zero diffusion, where we found that one eigenvalue is equal to zero whereas the others are concentrated at $A_{c}$ and 1 . The effect of diffusion is to shorten the plateaus where eigenvalues have real part 1 or $A_{c}$, and this behavior persists for the entire range of $A_{c}$ studied.

The continuous (fig. 10(a)) and piecewise (fig. 8) models display qualitatively similar features: the curves initially increase roughly quadratically with $k$ and are always positive. For larger $k$ values we find $\operatorname{Re}(\lambda) \sim k$, which suggests that for this $k$ regime the imaginary components of the evolution of the amplitudes dominate; and for still larger values of $k$ the dependence of $\operatorname{Re}(\lambda)(k)$ levels off. This feature occurs in the piecewise model at much larger $k$-values not shown in fig. 8 . In both cases smaller $\hat{D}$ gives rise to less stable eigenvalues. Precise comparison between the two models is difficult as the wave number $k$ is scaled with a factor $\alpha=20$ in the continuous case. This parameter $\alpha$ is necessary in order to find a stable shear banding solution in the continuous case [4]. One striking difference between the curves in fig. 8 and fig. 10(a) is the behavior in the limit $k \rightarrow 0$. In the smooth model with $k \rightarrow 0$ the smallest eigenvalue depends on $\hat{D}$. The eigenvalue 0 results only when $\hat{D} \rightarrow 0$, in contrast to the piecewise model which is always neutrally stable with respect to nonundulatory perturbations. This can mathematically be understood from the fact that the interface width scales as $\sqrt{\hat{D}}$ for $\hat{D} \rightarrow 0$. Therefore the matching at the interface can be done in a smoother fashion when $\hat{D}$ becomes increasingly smaller. In the limit $\hat{D} \rightarrow 0$ this will result in a solution to the piecewise model which approximates the solution to the smooth model. If we compare fig. 10(b) with fig. 9, 

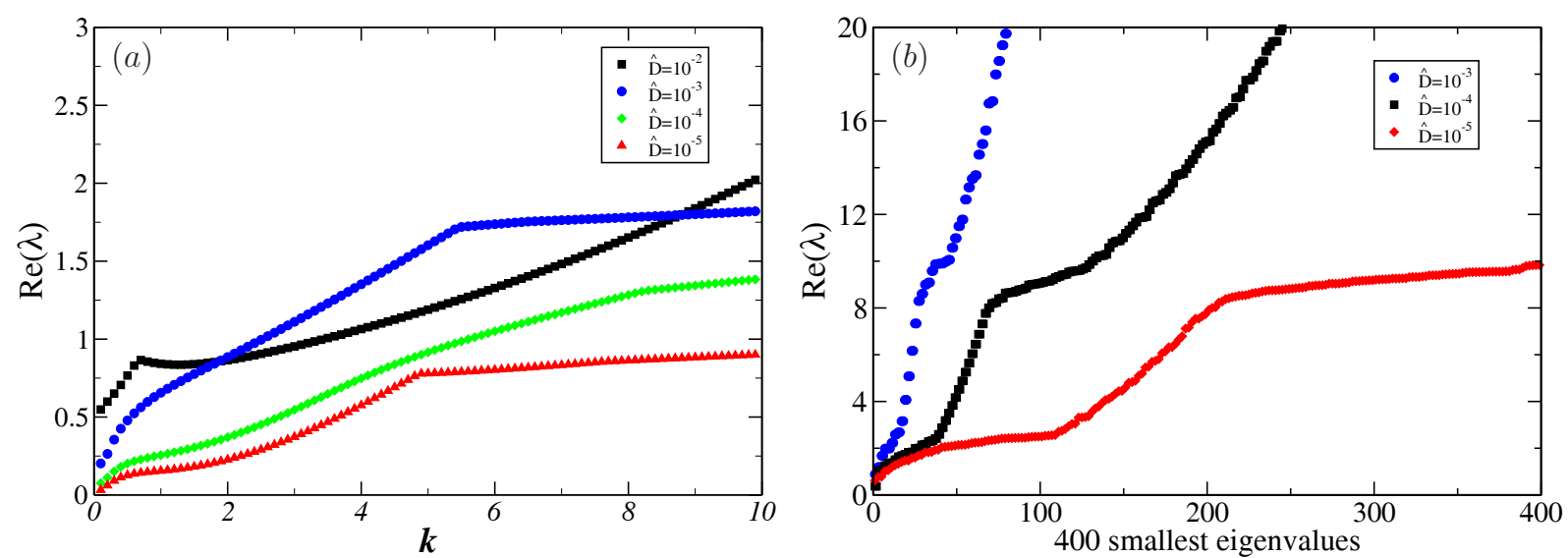

Fig. 10. Real part of the eigenvalue with the smallest real part, as a function of the undulation wave number $k$ for four different values of the diffusion constant $\hat{D}$ for the continuous model (a). For small $k<1$ the dependence of $\operatorname{Re}(\lambda)$ is again approximately quadratic. For larger $k$ bumps occur, which are probably caused by the fact that the imaginary parts of the equations start to dominate the eigenvalues. The distribution of 400 eigenvalues with smallest real part (b) for $k=3$ is very similar to the distribution obtained by the piecewise toy model of fig. 9 .

we see the same qualitative behavior. Again plateaus occur, but because of the smoothness of the model the curves are more regular than those obtained for the piecewise toy model. Nevertheless, fig. 10 suggests that the results for the piecewise model are generic and also valid for general smooth models without normal stresses.

From our findings we therefore conclude that normal stresses are generically responsible for rendering the Johnson-Segalman model linearly unstable for longwavelength undulations. This confirms the results which were first conjectured in refs. [14,20].

\section{Discussion}

We have studied, to a large extent analytically, a toy model for shear banding without normal stresses, but with spatial gradient terms (stress diffusion). We captured the general characteristics of a nonmonotonic stress-shear rate relation by introducing a piecewise linear stress-shear rate curve, which makes analytical calculations tractable. The results obtained for the piecewise model were shown to be in qualitative agreement with those obtained using a smooth function $g$.

For the set of parameters chosen, we found multiple stationary solutions when $\hat{D}<10^{-2}$. For $\hat{D}=10^{-3}$ we obtain three stationary states: one being the commonly observed two-band profile and the other two having three and four bands. The two-band profile was shown to be linearly stable with respect to two-dimensional undulations. The linear stability of the two-band profile is at variance with analogous results for Johnson-Segalman (JS) model, which was shown by Fielding to have linear instabilities for a certain range of $k$ vectors [18] with $k \approx 1$. This strongly suggests that normal stresses, absent in our model, are responsible for the linear instabilities arising in the JS model. Related recent work by Fielding and coworkers $[19,20]$ showed that these linear instabilities are suppressed if nonlinear effects are taken into account. It might be that nonlinear perturbations induce instabilities in the scalar model studied here. The behavior of such (weakly) nonlinear instabilities is a subject of interest and future research.

It is a pleasure to thank the British Council and NWO for financial assistance. This work is part of the research programme of the "Stichting voor Fundamenteel Onderzoek der Materie (FOM)", which is financially supported by the "Nederlandse Organisatie voor Wetenschappelijk Onderzoek (NWO)".

\section{Appendix A. Integration constants}

The integration constants $c_{1}, c_{2}, d_{1}, d_{2}$ can be expressed in terms of $\hat{y}_{0}$, the spatial position at which $\hat{\dot{\gamma}}$ equals $\hat{\dot{\gamma}}_{0}$. From the four equations (2.15-2.16), we find

$$
\begin{aligned}
c_{1}= & \frac{\hat{\dot{\gamma}}_{0}-\frac{\alpha \widehat{\Sigma}}{A_{c}}}{\cosh \left(\sqrt{\frac{A_{c}}{\hat{D}}} \hat{y}_{0}\right)}, \\
c_{2}= & \frac{\hat{\dot{\gamma}}_{1}-\alpha \widehat{\Sigma}}{\cosh \left[\left(\hat{y}_{1}-1\right) / \sqrt{\hat{D}}\right]}, \\
d_{1}= & \frac{\left(\alpha \widehat{\Sigma}-A_{c} \hat{\dot{\gamma}}_{0}\right) \cos \left(\sqrt{\frac{z}{\hat{D}}} \hat{y}_{0}\right)}{z} \\
d_{2}= & \frac{\tanh \left(\sqrt{\frac{A_{c}}{\hat{D}}} \hat{y}_{0}\right) \sin \left(\sqrt{\frac{z}{\hat{D}}} \hat{y}_{0}\right)\left(A_{c} \hat{\dot{\gamma}}_{0}-\alpha \widehat{\Sigma}\right)}{\sqrt{A_{c} z}}, \\
& +\frac{\tanh \left(\sqrt{\frac{A_{c}}{\hat{D}}} \hat{y}_{0}\right) \cos \left(\sqrt{\frac{z}{\hat{D}}} \hat{y}_{0}\right)\left(A_{c} \hat{\dot{\gamma}}_{0}-\alpha \widehat{\Sigma}\right)}{\left.\sqrt{\frac{z}{A_{c} z}} \hat{y}_{0}\right)}
\end{aligned}
$$




$$
\alpha \widehat{\Sigma}^{*}=\frac{A_{c}\left\{\hat{\dot{\gamma}}_{0}\left(A_{c}+z\right)\left(\hat{y}_{0}-\hat{y}_{1}\right)-\sqrt{\hat{D}}\left[\hat{\dot{\gamma}}_{0} \tanh \left(\sqrt{\frac{A_{c}}{\hat{D}}} \hat{y}_{0}\right)\left(\frac{A_{c}+z}{\sqrt{A_{c}}}\right)+\hat{\dot{\gamma}}_{1} \tanh \left(\frac{1-\hat{y}_{1}}{\sqrt{\hat{D}}}\right)(z+1)\right]+\langle\hat{\dot{\gamma}}\rangle z\right\}}{A_{c}\left(\hat{y}_{0}-\hat{y}_{1}+z-z \hat{y}_{1}\right)+\hat{y}_{0} z-\sqrt{\hat{D}}\left[\tanh \left(\sqrt{\frac{A_{c}}{\hat{D}}} \hat{y}_{0}\right)\left(\frac{A_{c}+z}{\sqrt{A_{c}}}\right)+A_{c}(1+z) \tanh \left(\frac{1-\hat{y}_{1}}{\sqrt{\hat{D}}}\right)\right]} .
$$

where $z=\frac{A_{c} \hat{\dot{\gamma}}_{0}-\hat{\dot{\hat{\gamma}}}_{1}}{\hat{\hat{\gamma}}_{1}-\hat{\dot{\gamma}}_{0}}$. From these equations, together with eqs. $(2.17,2.18)$, the values of $\hat{y}_{0}$ and $\hat{y}_{1}$ can in principle be determined as a function of $\widehat{\Sigma}$. To fix the value of the selected stress $\widehat{\Sigma}^{*}$ an additional equation is required. From the global constraint of an imposed average shear rate $\langle\hat{\dot{\gamma}}\rangle$ we can find an expression for $\widehat{\Sigma}^{*}$. We substitute eqs. (A.1) for the constants $c_{1,2}$ and $d_{1,2}$ into eqs. (2.14a$2.14 \mathrm{c}$ ), and calculate the average value of the shear rate directly by splitting up the integral in three parts: $\left[0, \hat{y}_{0}\right]$, $\left[\hat{y}_{0}, \hat{y}_{1}\right],\left[\hat{y}_{1}, 1\right]$. By equating the sum of the three parts to $\langle\hat{\gamma}\rangle$, we obtain the following expression for $\widehat{\Sigma}$ in terms of $\hat{y}_{0}, \hat{y}_{1},\langle\hat{\dot{\gamma}}\rangle$, and $\hat{\dot{\gamma}}_{0}, \hat{\dot{\gamma}}_{1}$ :

\section{see equation (A.2) above}

\section{Appendix B. Amplitude evolution equations}

The time evolution of the amplitudes $\alpha_{n}(k, \hat{t})$ and $\delta \hat{\dot{\gamma}}_{0}(k, \hat{t})$ can be determined by substituting $\delta \hat{\dot{\gamma}}^{\mathrm{I}}(\hat{x}, \hat{y}, \hat{t})$ in eq. (3.12a). When we project out the cosine modes and use the definition of the scaled amplitudes $\alpha_{n}(k, \hat{t})=$ $a_{n}(k, \hat{t})(2 n+1) \pi(-1)^{n} / 4$, we obtain

$$
\begin{aligned}
& \frac{\mathrm{d} \alpha_{m}}{\mathrm{~d} \hat{t}}-\left(\frac{\partial \hat{\dot{\gamma}}^{1 \mathrm{D}}}{\partial \hat{y}}\right)_{\hat{y}_{0}} \partial_{\hat{t}} \delta \hat{y}_{0}(k, \hat{t})-\partial_{\hat{t}} \alpha \delta \widehat{\Sigma}(k, \hat{t})= \\
& \left(A_{c}+\hat{D} k^{2}\right)\left(\frac{\partial \hat{\dot{\gamma}}^{1 \mathrm{D}}}{\partial \hat{y}}\right)_{\hat{y}_{0}} \delta \hat{y}_{0}(k, \hat{t}) \\
& -\left(A_{c}+\hat{D} k^{2}+\hat{D}\left(\frac{\pi(2 m+1)}{2 \hat{y}_{0}}\right)^{2}\right) \alpha_{m} \\
& +\hat{D} k^{2} \alpha \widehat{\Sigma}(k, \hat{t})-\frac{i k(-1)^{m}(2 m+1) \pi}{2 \hat{y}_{0}}\left[\sum_{n=1}^{\infty} \alpha_{n}(-1)^{n}\right. \\
& \times \frac{16 \hat{y}_{0}^{2}}{(2 n+1)^{3} \pi^{3}}\left[R_{m n}^{A}-T_{m}^{A}\right]+\left(\frac{\partial \hat{\dot{\gamma}}^{1 \mathrm{D}}}{\partial \hat{y}_{0}}\right)_{\hat{y}_{0}} \delta \hat{y}_{0}(k, \hat{t}) F_{m}^{A} \\
& +\sum_{n=1}^{\infty}\left[L_{m n}^{A}+\frac{D}{A_{c}} R_{m n}^{A}\right] \frac{4 \alpha_{n}(-1)^{n}}{(2 n+1) \pi}-\left(J_{m}^{A}+\frac{\hat{D}}{A_{c}} T_{m}^{A}\right) \\
& \left.\times\left[\alpha \delta \widehat{\Sigma}+\left(\frac{\partial \hat{\dot{\gamma}}^{1 \mathrm{D}}}{\partial \hat{y}}\right)_{\hat{y}_{0}} \delta \hat{y}_{0}(k, \hat{t})\right]\right] \text {. } \\
& 8 \sum_{n=0}^{\infty} \frac{\mathrm{d} \alpha_{n}}{\mathrm{~d} \hat{t}} \frac{1}{(2 n+1)^{2} \pi^{2}}-\alpha \partial_{\hat{t}} \delta \widehat{\Sigma}+\partial_{\hat{t}} \delta \hat{\gamma}_{0}= \\
& \hat{D} k^{2} \alpha \delta \widehat{\Sigma}-\left(A_{c}+\hat{D} k^{2}\right) \delta \hat{\gamma}_{0}-8\left(A_{c}+\hat{D} k^{2}\right) \\
& \times \sum_{n=0}^{\infty} \frac{\alpha_{n}}{(2 n+1)^{2} \pi^{2}}-\frac{2 \hat{D}}{\hat{y}_{0}^{2}} \sum_{n=0}^{\infty} \alpha_{n}
\end{aligned}
$$

$$
\begin{aligned}
& -\frac{i k}{\hat{y}_{0}}\left\{-\sum_{n=0}^{\infty} \frac{\alpha_{n}\left(1-(-1)^{n}\right) 16 \hat{y}_{0}^{2}}{(2 n+1)^{3} \pi^{3}} \hat{\dot{\gamma}}_{0}\right. \\
& +\sum_{n=0}^{\infty} \frac{8 \hat{y}_{0} \alpha_{n}}{(2 n+1)^{2} \pi^{2}}\left[\frac{\alpha \widehat{\Sigma} \hat{y}_{0}}{A_{c}}+\left(\hat{\dot{\gamma}}_{0}-\frac{\alpha \widehat{\Sigma}}{A_{c}}\right) \sqrt{\hat{D}}\right] \\
& -\alpha \delta \widehat{\Sigma}\left[\frac{\alpha \widehat{\Sigma} \hat{y}_{0}^{2}}{2 A_{c}}+\left(\hat{\dot{\gamma}}_{0}-\frac{\alpha \widehat{\Sigma}}{A_{c}}\right) \frac{\hat{D}}{A_{c}}\right] \\
& \left.+\hat{y}_{0} \delta \gamma_{0}\left(\frac{\alpha \hat{\Sigma}_{\hat{y}_{0}}}{A_{c}}+\left(\hat{\dot{\gamma}}_{0}-\frac{\alpha \widehat{\Sigma}}{A_{c}}\right) \sqrt{\hat{D}}\right)-\frac{\hat{y}_{0}^{2} \hat{\dot{\gamma}}_{0} \delta \gamma_{0}}{2}\right\} \equiv \\
& \hat{D} k^{2} \alpha \delta \widehat{\Sigma}-\left(A_{c}+\hat{D} k^{2}\right) \delta \hat{\gamma}_{0}-8\left(A_{c}+\hat{D} k^{2}\right) \\
& \times \sum_{n=0}^{\infty} \frac{\alpha_{n}}{(2 n+1)^{2} \pi^{2}}-\frac{2 \hat{D}}{\hat{y}_{0}^{2}} \sum_{n=0}^{\infty} \alpha_{n}-\frac{i k}{\hat{y}_{0}} \Pi_{1}(k, \hat{t}) .
\end{aligned}
$$

In eq. (B.2) we defined the real function $\Pi_{1}(k, \hat{t})$ as the imaginary contribution to the evolution of $\delta \hat{\gamma}_{0}$. The coefficients $R_{m n}^{A}, T_{m}^{A}, F_{m}^{A}, L_{m n}^{A}, J_{m}^{A}$ in eqs. (B.1) are defined in terms of integrals of functions on the interval $\left[0, \hat{y}_{0}\right]$ :

$$
\begin{aligned}
R_{m n}^{A}= & \int_{0}^{\hat{y}_{0}} \cos \left(\frac{(2 m+1) \pi \hat{y}}{2 \hat{y}_{0}}\right) \\
& \times \cos \left(\frac{(2 n+1) \pi \hat{y}}{2 \hat{y}_{0}}\right) \frac{\partial \hat{\dot{\gamma}}^{1 \mathrm{D}}}{\partial \hat{y}} \mathrm{~d} \hat{y}, \\
F_{m}^{A}= & \int_{0}^{\hat{y}_{0}} \cos \left(\frac{(2 m+1) \pi \hat{y}}{2 \hat{y}_{0}}\right) \frac{\hat{y}^{2}}{2} \frac{\partial \hat{\dot{\gamma}}^{1 \mathrm{D}}}{\partial \hat{y}} \mathrm{~d} \hat{y}, \\
L_{m n}^{A}= & \int_{0}^{\hat{y}_{0}} \cos \left(\frac{(2 m+1) \pi \hat{y}}{2 \hat{y}_{0}}\right) \\
& \times \cos \left(\frac{(2 n+1) \pi \hat{y}}{2 \hat{y}_{0}}\right) \frac{\alpha \widehat{\Sigma} \hat{y}}{A_{c}} \mathrm{~d} \hat{y},
\end{aligned}
$$

and $T_{m}^{A}=R_{m,-\frac{1}{2}}^{A}$, and $J_{m}^{A}=L_{m,-\frac{1}{2}}^{A}$. The evolution equations for the amplitudes $\delta_{n}(k, \hat{t})$ is derived by substituting the expression for $\delta \hat{\dot{\gamma}}^{\mathrm{III}}(\hat{x}, \hat{y}, \hat{t})$ in eq. (3.12c), projecting out the cosine modes and using the scaling relation between $d_{n}$ and $\delta_{n}$, yielding

$$
\begin{aligned}
& \frac{\mathrm{d} \delta_{m}}{\mathrm{~d} \hat{t}}-\left(\frac{\partial \hat{\dot{\gamma}}^{1 \mathrm{D}}}{\partial \hat{y}}\right)_{\hat{y}_{1}} \partial_{\hat{t}} \delta \hat{y}_{1}(k, \hat{t})-\partial_{\hat{t}} \alpha \delta \widehat{\Sigma}(k, \hat{t})= \\
& \left(1+\hat{D} k^{2}\right)\left(\frac{\partial \hat{\dot{\gamma}}^{1 \mathrm{D}}}{\partial \hat{y}}\right)_{\hat{y}_{1}} \delta \hat{y}_{1}(k, \hat{t}) \\
& -\left(1+\hat{D} k^{2}+\hat{D}\left(\frac{\pi(2 m+1)}{2\left(1-\hat{y}_{1}\right)}\right)^{2}\right) \delta_{m}
\end{aligned}
$$




$$
\begin{aligned}
& +\hat{D} k^{2} \alpha \widehat{\Sigma}(k, \hat{t})-\frac{i k(-1)^{m}(2 m+1) \pi}{2\left(1-\hat{y}_{1}\right)}\left[\sum_{n=1}^{\infty} \delta_{n}(-1)^{n}\right. \\
& \times \frac{16\left(1-\hat{y}_{1}\right)^{2}}{(2 n+1)^{3} \pi^{3}}\left[\tilde{R}_{m n}^{D}-\tilde{T}_{m}^{D}\right]+\left(\frac{\partial \hat{\dot{\gamma}}^{1 \mathrm{D}}}{\partial \hat{y}_{1}}\right)_{\hat{y}_{1}} \delta \hat{y}_{1}(k, \hat{t}) \tilde{F}_{m}^{D} \\
& +\sum_{n=1}^{\infty}\left[\tilde{L}_{m n}^{D}+\hat{D} \tilde{R}_{m n}^{D}\right] \frac{4 \delta_{n}(-1)^{n}}{(2 n+1) \pi}-\left(\tilde{J}_{m}^{D}+\hat{D} \tilde{T}_{m}^{D}\right) \\
& \left.\times\left[\alpha \widehat{\Sigma}+\left(\frac{\partial \hat{\dot{\gamma}}^{\mathrm{D}}}{\partial \hat{y}}\right)_{\hat{y}_{1}} \delta_{1}(k, \hat{t})\right]\right]-i k\left\langle\hat{\dot{\gamma}} \delta_{m}\right. \\
& +i k\left[\alpha \delta \widehat{\Sigma}+\left(\frac{\partial \hat{\dot{\gamma}}^{1 \mathrm{D}}}{\partial \hat{y}}\right)_{\hat{y}_{1}} \delta \hat{y}_{1}\right]\langle\hat{\dot{\gamma}}\rangle,
\end{aligned}
$$

where the functions $\tilde{R}_{m n}^{D}, \tilde{T}_{m}^{D}, \tilde{F}_{m}^{D}, \tilde{L}_{m n}^{D}, \tilde{J}_{m}^{D}$ are defined similarly to eqs. (B.3), with $\hat{y}_{0}$ everywhere replaced with $1-\hat{y}_{1}$ and $A_{c}$ set to 1 . If we integrate the amplitude equation for $d_{n}(k, t)$ from $\hat{y}_{1}$ to 1 , we obtain the evolution equation for the $\delta \hat{\dot{\gamma}}_{1}(k, \hat{t})$,

$$
\begin{aligned}
& 8 \sum_{n=0}^{\infty} \frac{\mathrm{d} \delta_{n}}{\mathrm{~d} \hat{t}} \frac{1}{(2 n+1)^{2} \pi^{2}}-\alpha \partial_{\hat{t}} \delta \widehat{\Sigma}+\partial_{\hat{t}} \delta \hat{\gamma}_{1}= \\
& \hat{D} k^{2} \alpha \delta \widehat{\Sigma}-\left(1+\hat{D} k^{2}\right) \delta \hat{\gamma}_{1}-8\left(1+\hat{D} k^{2}\right) \sum_{n=0}^{\infty} \frac{\delta_{n}}{(2 n+1)^{2} \pi^{2}} \\
& -\frac{2 \hat{D}}{\left(1-\hat{y}_{1}\right)^{2}} \sum_{n=0}^{\infty} \delta_{n} \\
& -\frac{i k}{\left(1-\hat{y}_{1}\right)}\left\{-\sum_{n=0}^{\infty} \frac{\delta_{n}\left(1-(-1)^{n}\right) 16\left(1-\hat{y}_{1}\right)^{2}}{(2 n+1)^{3} \pi^{3}}\right. \\
& +\sum_{n=0}^{\infty} \frac{8\left(1-\hat{y}_{1}\right) \delta_{n}}{(2 n+1)^{2} \pi^{2}}\left[\langle\hat{\gamma}\rangle+\alpha \widehat{\Sigma}\left(\hat{y}_{1}-1\right)-\left(\hat{\dot{\gamma}}_{1}-\alpha \widehat{\Sigma}\right) \sqrt{\hat{D}}\right] \\
& -\alpha \delta \widehat{\Sigma}\left[\langle\hat{\dot{\gamma}}\rangle\left(1-\hat{y}_{1}\right)-\frac{\alpha \widehat{\Sigma}\left(1-\hat{y}_{1}\right)^{2}}{2}-\left(\hat{\dot{\gamma}}_{1}-\alpha \widehat{\Sigma}\right) \hat{D}\right] \\
& -\left(1-\hat{y}_{1}\right) \delta \gamma_{1}\left(\langle\hat{\dot{\gamma}}\rangle-\alpha \widehat{\Sigma}\left(1-\hat{y}_{1}\right)+\left(\hat{\dot{\gamma}}_{1}-\alpha \widehat{\Sigma}\right) \sqrt{\hat{D}}\right) \\
& \left.+\frac{\left(1-\hat{y}_{1}\right)^{2} \hat{\dot{\gamma}}_{1} \delta \gamma_{1}}{2}\right\} \equiv \hat{D} k^{2} \alpha \delta \widehat{\Sigma}-\left(1+\hat{D} k^{2}\right) \delta \hat{\gamma}_{1} \\
& -8\left(1+\hat{D} k^{2}\right) \sum_{n=0}^{\infty} \frac{\delta_{n}}{(2 n+1)^{2} \pi^{2}}-\frac{2 \hat{D}}{\left(1-\hat{y}_{1}\right)^{2}} \sum_{n=0}^{\infty} \delta_{n} \\
& -\frac{i k}{\left(1-\hat{y}_{1}\right)} \Pi_{2}(k, \hat{t}),
\end{aligned}
$$

where $\Pi_{2}(k, \hat{t})$ is the imaginary contribution to the evolution of $\delta \hat{\gamma}_{1}$. The equation for the amplitudes $b_{n}(k, \hat{t})$ can be found from the evolution equation of $q$ (eq. (3.16)) and substituting expression (3.15), which leads to a lengthy differential equation for $b_{n}(k, \hat{t})$. After projecting out the sine modes over $\left[\hat{y}_{0}, \hat{y}_{1}\right]$, we find

$$
\begin{aligned}
& \frac{\mathrm{d} \beta_{m}}{\mathrm{~d} t}+\frac{1}{2}\left[(-1)^{m} \partial_{t}\left(\frac{\partial \hat{\dot{\gamma}}^{1 \mathrm{D}}}{\partial \hat{y}}\right)_{\hat{y}_{1}} \delta \hat{y}_{1}-\partial_{t}\left(\frac{\partial \hat{\dot{\gamma}}^{1 \mathrm{D}}}{\partial \hat{y}}\right)_{\hat{y}_{0}} \delta \hat{y}_{0}\right] \\
& -\frac{1}{2} \partial_{t} \alpha \delta \widehat{\Sigma}\left(1-(-1)^{m}\right)= \\
& \beta_{m}\left[z-\hat{D} k^{2}-\hat{D}\left(\frac{m \pi}{(2 M+1) \pi-h}\right)^{2}\right] \\
& +\frac{z-\hat{D} k^{2}}{2}\left[(-1)^{m}\left(\frac{\partial \hat{\dot{\gamma}}^{1 \mathrm{D}}}{\partial \hat{y}}\right)_{\hat{y}_{1}} \delta \hat{y}_{1}-\left(\frac{\partial \hat{\dot{\gamma}}^{1 \mathrm{D}}}{\partial \hat{y}}\right)_{\hat{y}_{0}} \delta \hat{y}_{0}\right] \\
& +\frac{1}{2} \hat{D} k^{2} \alpha \delta \widehat{\Sigma}\left(1-(-1)^{m}\right)
\end{aligned}
$$$$
-\frac{i k m \pi}{2\left(\hat{y}_{1}-\hat{y}_{0}\right)}\left\{-\left(\frac{\partial \hat{\dot{\gamma}}^{1 \mathrm{D}}}{\partial \hat{y}}\right)_{\hat{y}_{0}} \frac{\hat{y}_{0}^{2} \delta \hat{y}_{0}}{2}\left[-d_{1} P_{1}(m)+d_{2} P_{2}(m)\right]\right.
$$$$
+\hat{y}_{0} \delta \hat{y}_{0}\left(\frac{\partial \hat{\dot{\gamma}}^{1 \mathrm{D}}}{\partial \hat{y}}\right)_{\hat{y}_{0}}\left[-d_{1} V_{1}(m)+d_{2} V_{2}(m)\right]
$$$$
+\sum_{n=0}^{\infty} \frac{16 \alpha_{n} \hat{y}_{0}^{2}\left(1-(-1)^{n}\right)}{(2 n+1)^{3} \pi^{3}}\left[-d_{1} P_{1}(m)+d_{2} P_{2}(m)\right]
$$$$
-\sum_{n=0}^{\infty} \frac{8 \hat{y}_{0} \alpha_{n}}{(2 n+1)^{2} \pi^{2}}\left[-d_{1} V_{1}(m)+d_{2} V_{2}(m)+d_{1} \hat{y}_{0} P_{1}(m)\right.
$$$$
\left.-d_{2} \hat{y}_{0} P_{2}(m)\right]
$$$$
-\sum_{n=0}^{\infty} \frac{4 \beta_{n}\left(\hat{y}_{1}-\hat{y}_{0}\right)}{n^{2} \pi^{2}}\left[-d_{1} V_{1}(m)+d_{2} V_{2}(m)+d_{1} \hat{y}_{0} P_{1}(m)\right.
$$$$
\left.-d_{2} \hat{y}_{0} P_{2}(m)\right]+\sum_{n=1}^{\infty}\left[\frac{4 \beta_{n}\left(\hat{y}_{1}-\hat{y}_{0}\right)^{2}}{n^{3} \pi^{3}}-\frac{4 \hat{D} \beta_{n}}{\pi n z}\right]
$$$$
\times\left[-d_{1} G_{1}(m, n)+d_{2} G_{2}(m, n)\right]+\sum_{n=1}^{\infty} \frac{4 \beta_{n} Q_{1}(m, n)}{n \pi} \tilde{C}_{2}
$$$$
+\frac{-\hat{y}_{0}+(-1)^{m} \hat{y}_{1}}{m \pi}\left[\left(\frac{\partial \hat{\dot{\gamma}}^{1 \mathrm{D}}}{\partial \hat{y}}\right)_{\hat{y}_{1}} \delta \hat{y}_{1}-\left(\frac{\partial \hat{\dot{\gamma}}^{1 \mathrm{D}}}{\partial \hat{y}}\right)_{\hat{y}_{0}} \delta \hat{y}_{0}\right] \tilde{C}_{1}
$$$$
+\frac{1-\hat{y}_{0}+\left(\hat{y}_{1}-1\right)(-1)^{m} \hat{y}_{1}}{m \pi}\left[\hat{y}_{0}\left(\frac{\partial \hat{\dot{\gamma}}^{1 \mathrm{D}}}{\partial \hat{y}}\right)_{\hat{y}_{1}} \delta \hat{y}_{1}\right.
$$$$
\left.-\hat{y}_{1}\left(\frac{\partial \hat{\dot{\gamma}}^{1 \mathrm{D}}}{\partial \hat{y}}\right)_{\hat{y}_{0}} \delta \hat{y}_{0}\right] \tilde{C}_{1}-\tilde{C}_{2} W(m)
$$$$
\times\left[\left(\frac{\partial \hat{\dot{\gamma}}^{1 \mathrm{D}}}{\partial \hat{y}}\right)_{\hat{y}_{1}} \delta \hat{y}_{1}-\left(\frac{\partial \hat{\dot{\gamma}}^{1 \mathrm{D}}}{\partial \hat{y}}\right)_{\hat{y}_{0}} \delta \hat{y}_{0}\right]
$$$$
+\sqrt{\frac{\hat{D}}{z}}\left(\frac{1}{(2 M+1) \pi-h}\right)\left[d_{1} V_{1}(m)-d_{2} V_{2}(m)\right]
$$

$\times\left[\left(\frac{\partial \hat{\dot{\gamma}}^{1 \mathrm{D}}}{\partial \hat{y}}\right)_{\hat{y}_{0}} \delta \hat{y}_{0}-\left(\frac{\partial \hat{\dot{\gamma}}^{1 \mathrm{D}}}{\partial \hat{y}}\right)_{\hat{y}_{1}} \delta \hat{y}_{1}\right]$ 


$$
\begin{aligned}
& +\sqrt{\frac{\hat{D}}{z}}\left(\frac{1}{(2 M+1) \pi-h}\right)\left[d_{1} P_{1}(m)-d_{2} P_{2}(m)\right] \\
& \times\left[\left(\frac{\partial \hat{\dot{\gamma}}^{1 \mathrm{D}}}{\partial \hat{y}}\right)_{\hat{y}_{1}} \hat{y}_{0} \delta \hat{y}_{1}-\left(\frac{\partial \hat{\dot{\gamma}}^{1 \mathrm{D}}}{\partial \hat{y}}\right)_{\hat{y}_{0}} \hat{y}_{1} \delta \hat{y}_{0}\right] \\
& -\alpha \delta \widehat{\Sigma}\left(1-(-1)^{m}\right) \frac{\hat{y}_{1}-\hat{y}_{0}}{m \pi} \tilde{C}_{1} \\
& +\alpha \tilde{C}_{2}\left(\hat{y}_{1}-\hat{y}_{0}\right) \delta \widehat{\Sigma} \frac{\left(-\hat{y}_{0}+\hat{y}_{1}(-1)^{m}\right)}{m \pi} \\
& \left.-\alpha \delta \widehat{\Sigma}\left[d_{1} P_{1}(m)-d_{2} P_{2}(m)\right] \frac{\hat{D}}{z}\right\}-i k \tilde{C}_{1} \beta_{m},
\end{aligned}
$$

where

$$
\begin{aligned}
P_{1}(m)= & \sqrt{\frac{z}{\hat{D}}} \int_{\hat{y}_{0}}^{\hat{y}_{1}} \sin \left(\frac{m \pi\left(\hat{y}-\hat{y}_{0}\right)}{\hat{y}_{1}-\hat{y}_{0}}\right) \\
& \times \sin \left(\sqrt{\frac{z}{\hat{D}}} \hat{y}\right) \mathrm{d} \hat{y}, \\
V_{1}(m)= & \sqrt{\frac{z}{\hat{D}}} \int_{\hat{y}_{0}}^{\hat{y}_{1}} \sin \left(\frac{m \pi\left(\hat{y}-\hat{y}_{0}\right)}{\hat{y}_{1}-\hat{y}_{0}}\right) \\
& \times \sin \left(\sqrt{\frac{z}{\hat{D}}} \hat{y}\right) \hat{y} \mathrm{~d} \hat{y}, \\
G_{1}(m, n)= & \sqrt{\frac{z}{\hat{D}}} \int_{\hat{y}_{0}}^{\hat{y}_{1}} \sin \left(\frac{n \pi\left(\hat{y}-\hat{y}_{0}\right)}{\hat{y}_{1}-\hat{y}_{0}}\right) \sin \left(\frac{m \pi\left(\hat{y}-\hat{y}_{0}\right)}{\hat{y}_{1}-\hat{y}_{0}}\right) \\
& \times \sin \left(\sqrt{\left.\frac{z}{\hat{D}} \hat{y}\right) \hat{y} \mathrm{~d} \hat{y},}\right. \\
Q_{1}(m, n)= & \int_{\hat{y}_{0}}^{\hat{y}_{1}} \sin \left(\frac{n \pi\left(\hat{y}-\hat{y}_{0}\right)}{\hat{y}_{1}-\hat{y}_{0}}\right) \\
& \times \sin \left(\frac{m \pi\left(\hat{y}-\hat{y}_{0}\right)}{\hat{y}_{1}-\hat{y}_{0}}\right) \hat{y} \mathrm{~d} \hat{y}, \\
W(m)= & \int_{\hat{y}_{0}}^{\hat{y}_{1}} \sin \left(\frac{n \pi\left(\hat{y}-\hat{y}_{0}\right)}{\hat{y}_{1}-\hat{y}_{0}}\right) \frac{\hat{y}^{2}}{\hat{y}_{1}-\hat{y}_{0}} \mathrm{~d} \hat{y} .
\end{aligned}
$$

The functions $P_{2}(m), V_{2}(m), G_{2}(m, n)$ are obtained if the $\sin \left(\sqrt{\frac{z}{\hat{D}}} \hat{y}\right)$ function is replaced with $\cos \left(\sqrt{\frac{z}{\hat{D}}} \hat{y}\right)$. The constants $\tilde{C}_{1}$ and $\tilde{C}_{2}$ are defined by

$$
\begin{aligned}
\tilde{C}_{1}= & \frac{\alpha \widehat{\Sigma}-A_{c} \hat{\dot{\gamma}}_{0}}{z A_{c}} \hat{y}_{0}\left(z+A_{c}\right)+\sqrt{\frac{\hat{D}}{A_{c}}}\left(\hat{\dot{\gamma}}_{0}-\frac{\alpha \widehat{\Sigma}}{A_{c}}\right) \\
& -\sqrt{\frac{\hat{D}}{z}}\left[d_{1} \sin \left(\sqrt{\frac{z}{\hat{D}}} \hat{y}_{0}\right)-d_{2} \cos \left(\sqrt{\frac{z}{\hat{D}}} \hat{y}_{0}\right)\right], \\
\tilde{C}_{2}= & \hat{\dot{\gamma}}_{0}+\frac{A_{c} \hat{\dot{\gamma}}_{0}-\alpha \hat{\Sigma}}{z} .
\end{aligned}
$$

The final evolution equation we need is that for $\delta \widehat{\Sigma}$, which is obtained by using eqs. (B.2) and (B.5) and substituting the expressions for $\partial_{t} \delta \hat{\gamma}_{0}, \partial_{t} \delta \hat{\gamma}_{1}$ in the differentiated eq. (3.18). We then find that the evolution equations for the average stress perturbation $\delta \widehat{\Sigma}$ read

$$
\begin{aligned}
& \alpha \partial_{\hat{t}} \delta \widehat{\Sigma}-4\left(\hat{y}_{1}-\hat{y}_{0}\right) \sum_{n=0}^{\infty} \frac{\mathrm{d} \alpha_{n}}{\mathrm{~d} t} \frac{1}{(2 n+1)^{2} \pi^{2}}-4\left(\hat{y}_{1}-\hat{y}_{0}\right) \\
& \times \sum_{n=0}^{\infty} \frac{\mathrm{d} \delta_{n}}{\mathrm{~d} t} \frac{1}{(2 n+1)^{2} \pi^{2}}+8\left(\hat{y}_{1}-\hat{y}_{0}\right) \\
& \times \sum_{n=0}^{\infty} \frac{\mathrm{d} \beta_{2 n+1}}{\mathrm{~d} \hat{t}} \frac{1}{(2 n+1)^{2} \pi^{2}}= \\
& +4\left(1+\hat{D} k^{2}\right)\left(2-\hat{y}_{1}-\hat{y}_{0}\right) \sum_{n=0}^{\infty} \frac{\delta_{n}}{(2 n+1)^{2} \pi^{2}} \\
& +4\left(A_{c}+\hat{D} k^{2}\right)\left(\hat{y}_{1}+\hat{y}_{0}\right) \sum_{n=0}^{\infty} \frac{\alpha_{n}}{(2 n+1)^{2} \pi^{2}}+\frac{\hat{D}\left(\hat{y}_{0}+\hat{y}_{1}\right)}{\hat{y}_{0}^{2}} \\
& \times \sum_{n=0}^{\infty} \frac{\alpha_{n}}{(2 n+1)^{2} \pi^{2}} \\
& +\frac{\hat{D}\left(2-\hat{y}_{0}-\hat{y}_{1}\right)}{\left(1-\hat{y}_{1}\right)^{2}} \sum_{n=0}^{\infty} \frac{\delta_{n}}{(2 n+1)^{2} \pi^{2}}+\left(1+\hat{D} k^{2}\right) \\
& \times\left(2-\hat{y}_{1}-\hat{y}_{0}\right) \frac{\delta \hat{\gamma}_{1}}{2}+\left(A_{c}+\hat{D} k^{2}\right)\left(\hat{y}_{1}+\hat{y}_{0}\right) \frac{\delta \hat{\gamma}_{0}}{2}-\alpha \hat{D} k^{2} \delta \hat{\Sigma} \\
& +i k\left(\frac{\left(\hat{y}_{0}+\hat{y}_{1}\right)}{2 \hat{y}_{0}} \Pi_{1}(k, \hat{t})+\frac{2-\hat{y}_{1}-\hat{y}_{0}}{2\left(1-\hat{y}_{1}\right)} \Pi_{2}(k, \hat{t})\right) . \quad(\mathrm{B} .9)
\end{aligned}
$$

\section{Appendix C. Continuous model}

In this appendix we present the results of stability analysis of the first model, in which the nonmonotonic stress is encoded in the smooth function $g(\xi)$ (eq. (2.3)). A straightforward calculation shows that for this case the amplitudes $a_{n}(k, \hat{t})$ are not given by eq. (B.1), but instead obey

$$
\begin{aligned}
& \sum_{n=0}^{\infty} \frac{\mathrm{d} a_{n}(k, \hat{t})}{\mathrm{d} \hat{t}} \cos (n \pi \hat{y})=-\sum_{n=0}^{\infty}\left[1+\hat{D}\left(n^{2} \pi^{2}+k^{2}\right)\right] \\
& \times a_{n}(k, \hat{t}) \cos (n \pi \hat{y})-\alpha g^{\prime}\left[\alpha\left(\left\langle\hat{\sigma}_{p}^{1 \mathrm{D}}\right\rangle+\langle\hat{\gamma}\rangle / \alpha-\hat{\sigma}_{p}^{1 \mathrm{D}}(y)\right)\right] \\
& \times \sum_{n=1}^{\infty} a_{n}(k, \hat{t}) \cos (n \pi \hat{y}) \\
& +i k\left\{\alpha \sum_{n=1}^{\infty} a_{n}(k, \hat{t})\left[\frac{\cos (n \pi \hat{y})-1}{n^{2} \pi^{2}}\right] \partial_{\hat{y}} \hat{\sigma}_{p}^{1 \mathrm{D}}(\hat{y})\right. \\
& \left.-\sum_{n=0}^{\infty} a_{n}(k, \hat{t}) \hat{v}^{1 \mathrm{D}}(\hat{y}) \cos (n \pi \hat{y})\right\} .
\end{aligned}
$$

Projecting out the cosines, we have the following matrix equation:

$$
\dot{\mathbf{a}}=-\left(\mathbf{P}^{\mathrm{cont}} \cdot \mathbf{a}-2 i \alpha k \mathbf{Q}^{\mathrm{cont}} \cdot \mathbf{a}\right),
$$




$$
\begin{aligned}
& Q_{00}^{\mathrm{cont}}=-\frac{\langle\hat{\dot{\gamma}}\rangle}{4 \alpha}+\frac{\left\langle\hat{\sigma}_{p}^{1 \mathrm{D}}\right\rangle}{4}-\frac{1}{2} \int_{0}^{1} y \hat{\sigma}_{p}^{1 \mathrm{D}}(y) \mathrm{d} y, \\
& Q_{0 n}^{\mathrm{cont}}= \begin{cases}\frac{\left\langle\hat{\sigma}_{p}^{1 \mathrm{D}}\right\rangle+\frac{\langle\hat{\gamma}\rangle}{\alpha}-\hat{\sigma}_{p}^{1 \mathrm{D}}(1)}{n^{2} \pi^{2}} & (n \text { odd }), \\
0 & (n \text { even }),\end{cases} \\
& Q_{n 0}^{\text {cont }}= \begin{cases}\frac{2\left(\left\langle\hat{\sigma}_{p}^{1 \mathrm{D}}\right\rangle+\frac{\langle\hat{\gamma}\rangle}{\alpha}\right)}{n^{2} \pi^{2}}-\int_{0}^{1} \hat{\sigma}_{p}^{1 \mathrm{D}}(y) \frac{\sin (n \pi y)}{n \pi} \mathrm{d} y & (n \text { odd }), \\
-\int_{0}^{1} \hat{\sigma}_{p}^{1 \mathrm{D}}(y) \frac{\sin (n \pi y)}{n \pi} \mathrm{d} y & (n \text { even }),\end{cases} \\
& Q_{n n}^{\mathrm{cont}}=-\frac{1}{4}\left(\frac{\langle\gamma\rangle}{\alpha}+\left\langle\hat{\sigma}_{p}^{1 \mathrm{D}}\right\rangle\right)+\frac{3}{4 n \pi} \int_{0}^{1} \hat{\sigma}_{p}^{1 \mathrm{D}}(y) \sin (2 \pi n y) \mathrm{d} y \\
& -\frac{1}{2} \int_{0}^{1} \hat{\sigma}_{p}^{1 \mathrm{D}}(y) y \mathrm{~d} y-\frac{1}{n \pi} \int_{0}^{1} \sin (n \pi y) \hat{\sigma}_{p}^{1 \mathrm{D}}(y) \mathrm{d} y+\left(\frac{1-(-1)^{n}}{n^{2} \pi^{2}}\right) \hat{\sigma}_{p}^{1 \mathrm{D}}(1) \quad(n \geq 1), \\
& Q_{m n}^{\mathrm{cont}}=-\left(\frac{\langle\hat{\dot{\gamma}}\rangle}{\alpha}+\left\langle\hat{\sigma}_{p}^{1 \mathrm{D}}\right\rangle\right)\left(\frac{-2 m^{2}-2 n^{2}+(-1)^{m-n}(m+n)^{2}+(-1)^{m+n}(m-n)^{2}}{2 \pi^{2}(m-n)^{2}(m+n)^{2}}\right) \\
& -\int_{0}^{1} \frac{\hat{\sigma}_{p}^{1 \mathrm{D}}(y)[m \sin ((n-m) \pi y)+n \sin ((n-m) \pi y)-m \sin ((n+m) \pi y)+n \sin ((n+m) \pi y)]}{2 \pi^{2}\left(n^{2}-m^{2}\right)} \\
& +\int_{0}^{1}\left[\frac{\sin (n \pi y) \cos (m \pi y)}{n \pi}\right] \hat{\sigma}_{p}^{1 \mathrm{D}}(y) \mathrm{d} y+(-1)^{m} \hat{\sigma}_{p}^{1 \mathrm{D}}(1)\left[\frac{(-1)^{n}-1}{n^{2} \pi^{2}}\right] \\
& +\int_{0}^{1} \hat{\sigma}_{p}^{1 \mathrm{D}}(y)\left[\frac{\cos (n \pi y)-1}{n^{2} \pi^{2}}\right] m \pi \sin (m \pi y) \mathrm{d} y \quad(m \neq n, m, n \geq 1) .
\end{aligned}
$$

where the dot denotes denotes differentiation with respect to time. The entries of matrix $\mathbf{P}^{\text {cont }}$ are given as

$$
\begin{aligned}
P_{00}^{\text {cont }}= & \left(1+\hat{D} k^{2}\right), \\
P_{0 n}^{\text {cont }}= & \alpha \int_{0}^{1} g^{\prime}\left[\alpha\left(\left\langle\hat{\sigma}_{p}^{1 \mathrm{D}}\right\rangle+\langle\hat{\dot{\gamma}}\rangle / \alpha-\hat{\sigma}_{p}^{1 \mathrm{D}}(y)\right)\right] \\
& \times \cos (n \pi y) \mathrm{d} y \quad(n \geq 1), \\
P_{n 0}^{\mathrm{cont}}= & 0 \quad(n \geq 1), \\
P_{m n}^{\mathrm{cont}}= & \left(1+\hat{D}\left(n^{2} \pi+k^{2}\right)\right) \delta_{n m}+2 \alpha \int_{0}^{1} g^{\prime}\left[\alpha \left(\left\langle\hat{\sigma}_{p}^{1 \mathrm{D}}\right\rangle\right.\right. \\
& \left.\left.+\langle\hat{\dot{\gamma}}\rangle / \alpha-\hat{\sigma}_{p}^{1 \mathrm{D}}(y)\right)\right] \cos (n \pi y) \\
& \times \cos (m \pi y) \mathrm{d} y \quad(m, n \geq 1) .
\end{aligned}
$$

For the matrix $\mathbf{Q}^{\text {cont }}$, we find the entries

$$
\text { see equations (C.7) to (C.11) above }
$$

All the matrix elements have to be computed numerically, after which the linear stability of the solutions can be examined along the same lines as for the piecewise model. From these computations, we conclude that the continuous equivalent of the piecewise system exhibits the same stability behavior as the piecewise model: it is linearly stable. For long-wavelength undulations the dispersion relation is again dominated by the real diagonal matrix elements. That is, we find again a quadratic dependence of the eigenvalue with the smallest real part on $k$.

\section{References}

1. I. Cohen, B. Davidovitch, A. Schofield, M. Brenner, D. Weitz, Phys. Rev. Lett. 97, 215502 (2006).

2. V. Schmitt, F. Lequeux, A. Pousse, D. Roux, Langmuir 10, 955 (1994).

3. O. Radulescu, P.D. Olmsted, Rheol. Acta 38, 606 (1999).

4. N.A. Spenley, X.F. Yuan, M.E. Cates, J. Phys. II 6, 551 (1996).

5. C.Y.D. Lu, P.D. Olmsted, R.C. Ball, Phys. Rev. Lett. 84, $642(2000)$.

6. M. Doi, S.F. Edwards, The Theory of Polymer Dynamics (Clarendon, Oxford, 1989).

7. T. Shimada, M. Doi, K. Okano, J. Phys. Soc. Jpn. 57, 2432 (1988).

8. M.E. Cates, J. Phys.: Condens Matter 8, 9167 (1996).

9. M.R. Lopez-Gonzalez, W.M. Holmes, P.T. Callaghan, P.J. Photinos, Phys. Rev. Lett. 93, 268302 (2004).

10. S. Lerouge, J.P. Decruppe, J.F. Berret, Langmuir 16, 6464 (2000).

11. L. Becu, S. Manneville, A. Colin, Phys. Rev. Lett. 93 018301 (2004).

12. M.E. Cates, D.A. Head, A. Ajdari, Phys. Rev. E 66, 025202 (2002).

13. A. Aradian, M.E. Cates, Europhys. Lett. 70, 397 (2005).

14. S.M. Fielding, P.D. Olmsted, Phys. Rev. Lett. 92, 084502 (2004).

15. R. Ganapathy, A. Sood, Phys. Rev. Lett. 96, 108301 (2006).

16. R. Ganapathy, A. Sood, Langmuir 22, 11016 (2006).

17. R. Bandyopadhyay, G. Basappa, A.K. Sood, Phys. Rev. Lett. 84, 2022 (2000).

18. S.M. Fielding, Phys. Rev. Lett. 95, 134501 (2005). 
19. S.M. Fielding, P.D. Olmsted, Phys. Rev. Lett. 96, 104502 (2006).

20. H. Wilson, S. Fielding, J. Non-Newt. Fluid Mech. 138, 181 (2006)

21. P.D. Olmsted, O. Radulescu, C.-Y.D. Lu, J. Rheol. 44, 257 (2000).

22. W. Press, S.A. Teukolsky, W.T. Vetterling, B.P. Flannery, Numerical Recipes in $C$, 2nd edition (Cambridge University Press, Cambridge, 1992).
23. P. Grindrod, The Theory and Applications of ReactionDiffusion Equations Patterns and Waves (Clarendon Press, Oxford, 1996).

24. C.-S. Yih, J. Fluid Mech. 29, 539 (1967).

25. E. Anderson, Z. Bai, C. Bischof, S. Blackford, J. Demmel, J. Dongarra, J. Du Croz, A. Greenbaum, S. Hammarling, A. McKenney, D. Sorensen, LAPACK Users' Guide (Society for Industrial and Applied Mathematics, Philadelphia, PA, 1999). 